\title{
Oxetane Ethers are Formed Reversibly in the Lithium-Catalyzed Friedel-Crafts Alkylation of Phenols with Oxetanols: Synthesis of Dihydrobenzofurans, Diaryloxetanes, and Oxetane Ethers
}

\author{
Rosemary A. Croft ${ }^{[\mathrm{a}]}$, James J. Mousseau ${ }^{[\mathrm{b}]}$, Chulho Choi ${ }^{[\mathrm{b}]}$ and James A. Bull ${ }^{[\mathrm{a}]_{*}}$ \\ ${ }^{[a]}$ Department of Chemistry, Imperial College London, South Kensington, London, SW7 2AZ, UK. \\ ${ }^{[b]}$ Pfizer Medicine Design, Eastern Point Road, Groton, CT 06340, United States
}

To the late Sir Derek

\section{ARTICLE INFO}

\section{ABSTRACT}

\section{Article history:}

Received

Received in revised form

Accepted

Available online

Keywords:

Oxetanes;

Dihydrobenzofurans;

O-Alkylation;

Lithium;

Catalysis;

\begin{abstract}
Studies on the mechanism and intermediate products in the Friedel-Crafts reaction between oxetanols and phenols are presented. Formation of $O$-alkylated intermediates is identified using ${ }^{1} \mathrm{H}$ NMR spectroscopy, in a reversible formation of the kinetic oxetane ether products. An interesting relationship between the electronic nature of the nucleophile and the degree of $O$ alkylation is uncovered. For phenols substituted with an electron withdrawing group such as $\mathrm{CN}$, oxetane ethers are the only products isolated regardless of reaction time. Increasing the electron rich nature of the phenol leads to an increased proportion of the thermodynamic $C$-alkylated Friedel-Crafts products after just one hour and as the sole product/s after extended reaction times. These studies have enabled a more complete catalytic cycle to be proposed. Using the same lithium catalyst and carefully selected reaction times, several examples of oxetane ethers are successfully isolated as novel bioisosteres for ester groups.
\end{abstract}

2009 Elsevier Ltd. All rights reserved.

\section{Introduction}

The Friedel-Crafts alkylation reaction was first reported in 1877 and has remained a powerful approach for the formation of alkylated aromatic compounds (Figure 1A). ${ }^{[1]}$ The generation of a carbocation can be effected through the use of powerful Lewis acids, traditionally from secondary or tertiary halides. In the lifetime of the carbocation, competing rearrangement reactions can occur to generate more stable carbocations, so alternative approaches such as acylation followed by reduction can be appropriate in some cases. Recently, catalytic Friedel-Crafts reactions have been reported, using various Lewis acid catalysts, and importantly from alcohols as substrates. ${ }^{[2,3]}$ This process produces only water as a stoichiometric side product, under much milder conditions. Electron rich aromatic derivatives, including phenols, are often required for reactivity. The generation of carbocations has also been employed for the synthesis of ethers, using oxygen nucleophiles, often in alcohol solvents. However, in Friedel-Crafts reactions with phenols, the corresponding ether resulting from nucleophilic attack with the oxygen atom is rarely reported (Figure 1B). ${ }^{[4]}$ The effect of temperature and reaction time on ether formation in FriedelCrafts reactions has been investigated in separate prior studies by Poliakoff and Adam. ${ }^{[5,6]}$ In general, higher temperatures and longer reaction times were shown to favor $C$-alkylation over
$O$-alkylation. However, to date, the effect of the electronic nature of the nucleophile remains unexplored.

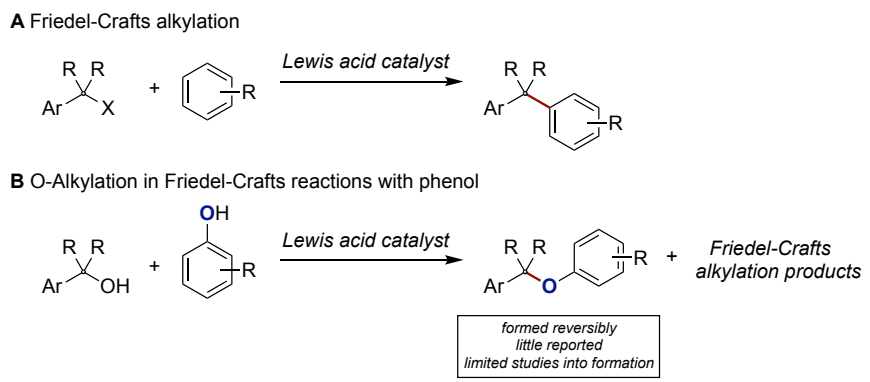

Figure 1. Friedel Crafts alkylation and O-alkylation in FriedelCrafts reactions with phenols.

Recent years has seen significant interest in the use of oxetanes as synthetic intermediates, and as replacement groups in medicinal chemistry. ${ }^{[7,8,9]}$ Particular interest has arisen through the use of oxetanes as polar replacement groups with improved metabolic stability for gem-dimethyl ${ }^{[10]}$ or tert-butyl groups, ${ }^{[11]}$ and as bioisosteres for ketones, ${ }^{[12,13]}$ amides, ${ }^{[14]}$ thioesters, ${ }^{[15]}$ and carboxylic acids ${ }^{[16]}$ (Figure 2A). These bioisosteric replacements can afford improvements in physicochemical properties and increased options for medicinal chemists. The ring opening of oxetanes has also been applied in the synthesis of varied other 
heterocyclic derivatives, themselves important pharmacophores. $^{[17]}$

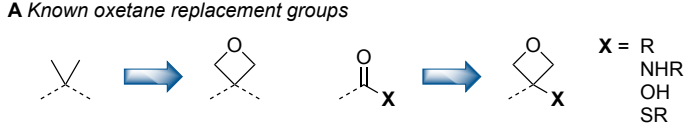

B This work:

$$
{ }_{\mathrm{Ar}} \mathrm{N}_{\mathrm{OR}} \longrightarrow \mathrm{Ar}_{\mathrm{OR}}^{\mathrm{O}} \text { 。 New chemical space }
$$

Figure 2. A) Oxetanes can act as bioisosteres for gem-dimethyl groups, and the carbonyl group in ketones, amides, carboxylic acids and thioesters. B) Oxetane ethers are proposed as interesting replacement groups for esters.

Recently, we reported the synthesis of diaryloxetanes through a Li-catalyzed Friedel-Crafts alkylation reaction with phenol, using 3-aryl-oxetan-3-ols as substrates (Scheme 1). ${ }^{[13]}$ Using ortho-substituted phenols the oxetane derivatives were formed as the major products ( $>95 \%$ selective). On the other hand, with para-substituted phenols, the diaryloxetane was not observed, instead a dihydrobenzofuran product was observed. Interestingly reactions involving para-substituted phenols generally gave higher yields of the dihydrobenzofuran compared to the isomeric diaryloxetanes, but required prolonged reaction $(20 \mathrm{~h}$, Scheme 1B). Using phenol itself, the diaryloxetane and dihydrobenzofuran were formed in a $3: 1$ ratio, consistent with $o: p$ ratios often observed in Friedel-Crafts reactions. The dihydrobenzofuran product was assumed to result from a Friedel-Crafts reaction at the ortho-position of the phenol, followed by rapid intramolecular ring opening of the oxetane with the phenolic $\mathrm{OH}$ group.

A
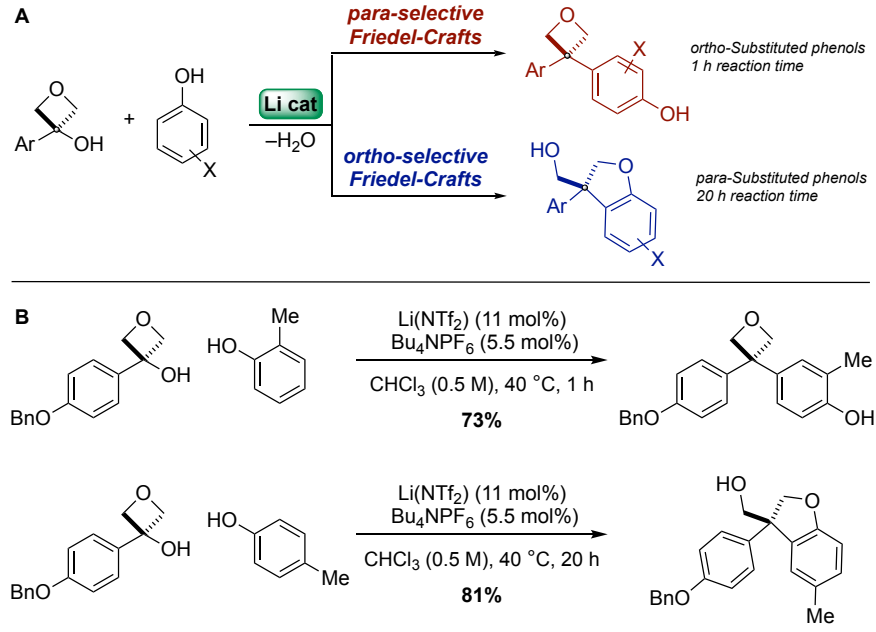

Scheme 1. A divergent Friedel-Crafts reaction between oxetanols and phenol.

Here we report further studies into this process, and provide a more complete mechanistic picture of the formation of the dihydrobenzofuran motifs. In situ ${ }^{1} \mathrm{H}$ NMR studies illustrate the progress of the reaction. Notably, oxetane ethers, formed by $O$-alkylation of the phenol nucleophiles are identified, and shown to be off-cycle species. These constitute rare examples of $O$-linked Friedel-Crafts products. The off-cycle equilibrium appears to aid in stabilizing the proposed oxetane carbocationic intermediate, and enhancing the yield of the dihydrobenzofuran product compared to the diaryloxetane derivatives formed with ortho-substituted phenols. The effects of the electronic nature of the phenols on the reaction are reported, with different product types formed depending on the para-substituent. With careful choice of reaction time, oxetane ether products can be isolated from even electron rich examples.

\section{Results and Discussion}

\subsection{Formation of oxetane ethers}

While attempting to extend our protocol towards further phenol derivatives with electron poor substituents, we were fascinated to observe a third product class from this reaction type. Using oxetanol 1 and 4-cyanophenol 2a, the $O$-alkylated product 3a was isolated as the only product after both 1 and $20 \mathrm{~h}$, exemplifying this unusual reactivity. The electron-poor nature of the phenol prevented Friedel-Crafts alkylation, but the $\mathrm{OH}$ was still a viable nucleophile to react with the oxetane carbocation.

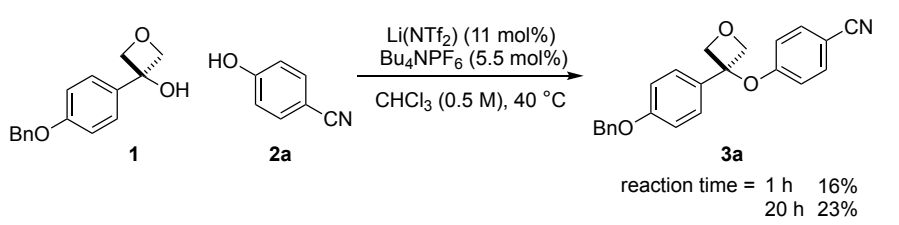

Scheme 2. Reaction using 4-cyanophenol under Li-catalyzed alkylation conditions forming ether $\mathbf{3 a}$.

Oxetane 3a represents the first example of such an oxetane aryl ether motif, which was interesting as a potential bioisostere for the corresponding ester group. The oxetane polarity and lone pair arrangement suitably mimic that of the ester carbonyl group (Figure 2B). Therefore, with these considerations in mind, we looked in more detail at the electronic factors of the phenol and the effect on the product/s formed in the reaction, aiming to provide more insight into the reaction mechanism and observe possible intermediates.

Initial studies took a range of phenols $\mathbf{2 a - f}$ with varying parasubstituents and examined the product distribution after $1 \mathrm{~h}$ and $20 \mathrm{~h}$ (Figure 3). An increase in combined yield was obtained as the electron donating ability of the para-substituent increased. Halogenated substituents; I, Cl and F, all gave the oxetane ether products $\mathbf{3 b}$-d as the major component in the reaction mixture after $1 \mathrm{~h}$. The dihydrobenzofuran was also observed in small quantities in all 3 cases alongside trace amounts of starting material. After $20 \mathrm{~h}$, the only product observed was the dihydrobenzofuran from Friedel-Crafts alkylation and intramolecular oxetane ring opening. The barrier to the formation of oxetane ether 3 is evidently lower than for dihydrobenzofuran 4 and ether 3 appeared to be formed rapidly and reversibly as a kinetic product. Dihydrobenzofuran $\mathbf{4}$ is formed irreversibly as the thermodynamically preferred product.

The reactivity of phenols with more electron rich substituents was greatly increased towards products involving dihydrobenzofuran 4. Both 4-methylphenol 2e and 4-methoxyphenol $2 \mathrm{f}$ gave dihydrobenzofuran $\mathbf{4}$ as the main product in high yields after $1 \mathrm{~h}$ as well as small quantities of the oxetane ether product $(<11 \%)$. A second product was also observed in significant quantities in these reactions and was identified as dimer $\mathbf{5}$ formed through addition of the primary alcohol of the dihydrobenzofuran to a further equivalent of the oxetanyl carbocation that is generated in situ. This rapid formation of dihydrobenzofuran $\mathbf{4}$ and dimer 5 show that the energy barrier for formation is lower with electron rich parasubstituted phenols compared to electron withdrawing halogen or $\mathrm{CN}$ substituents. After $20 \mathrm{~h}$, the only product observed was dihydrobenzofuran $\mathbf{4}$, indicating the dimer formation was also reversible. 

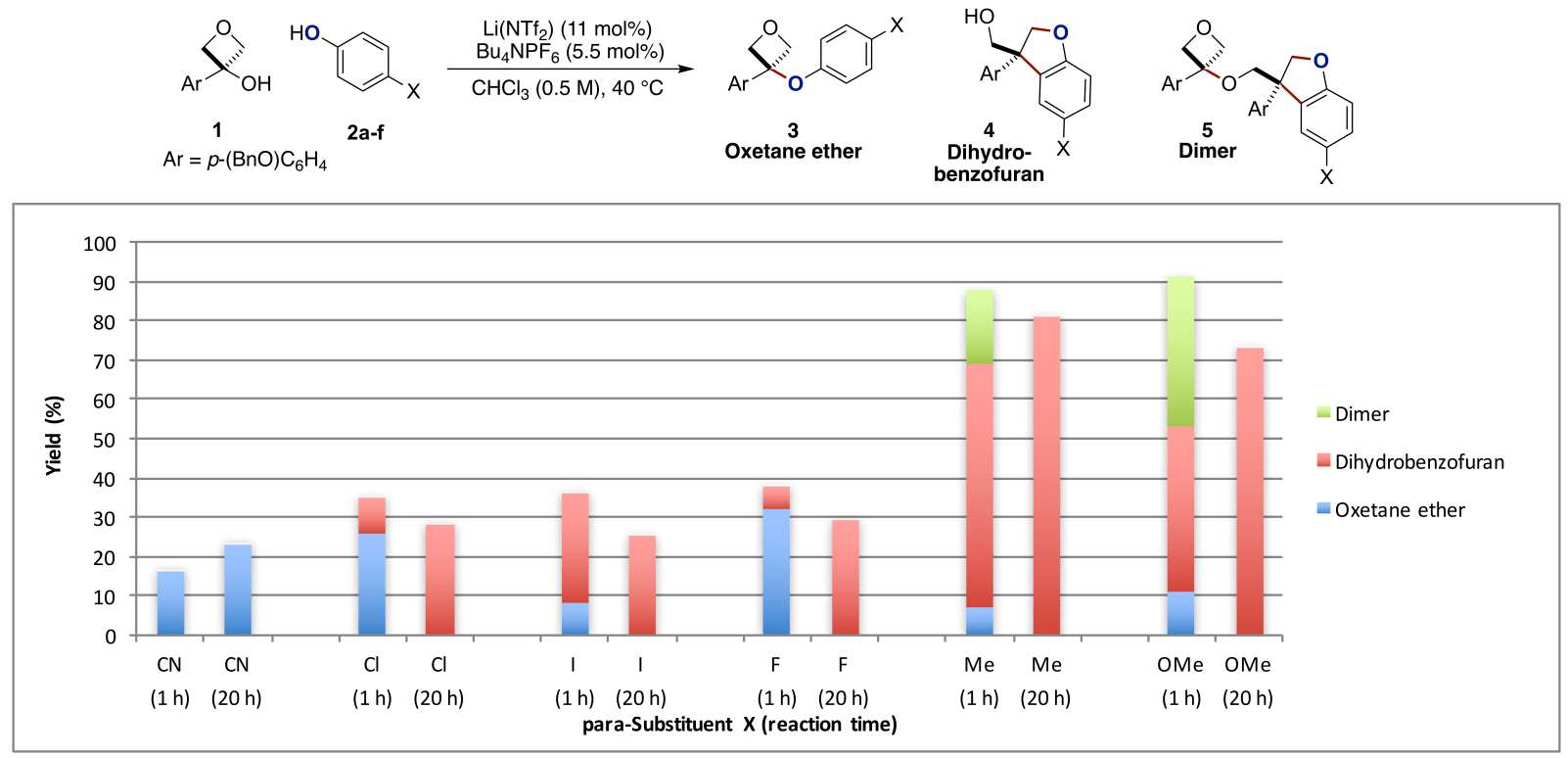

Figure 3. Product distribution (isolated yields) of the reaction of para-substituted phenols $\mathbf{2}$ with oxetanol $\mathbf{1}$ after $1 \mathrm{~h}$ and $20 \mathrm{~h}$. New bonds formed highlighted in red.

\section{2. ${ }^{1} H$ NMR studies to monitor reaction progress in situ}

For a deeper understanding of the formation and reversibility of the ether products, selected reactions were studied by ${ }^{1} \mathrm{H}$ NMR spectroscopy. Reactions were conducted in NMR tubes and monitored every 30 seconds for the first hour and every 5 minutes thereafter until there was no further change in the reaction composition. Mesitylene was used as an internal standard in all the ${ }^{1} \mathrm{H}$ NMR studies as it was shown not to interfere with the desired reactivity. There was a notable solubility difference in the NMR tube compared to the reactions previously conducted in round bottom flasks. The catalyst dissolved almost instantaneously in the NMR tube whereas in a round bottom flask or microwave vial solubilization of the catalyst took up to $20 \mathrm{~min}$ indicated by the initial cloudy suspension becoming clear. This may be a result of a greater surface to volume ratio and extra moisture associated with the glassware when using the ${ }^{1} \mathrm{H}$ NMR tube. However, the result was that the reactions conducted in NMR tubes proceeded more rapidly in the early stages.

The reaction between oxetanol $\mathbf{1}$ and 4-fluorophenol $\mathbf{2 d}$ was examined first. Generation of the oxetanyl carbocation was rapid under the reaction conditions with a maximal yield of the oxetane ether 3d $(64 \%)$ observed after just 4 minutes. Subsequently the quantity of oxetane ether $\mathbf{3 d}$ decreased steadily until the reaction reached completion at approximately $18 \mathrm{~h}$. The amount of dihydrobenzofuran $\mathbf{4 d}$ increased steadily throughout the reaction and was the sole product at the end of the reaction.

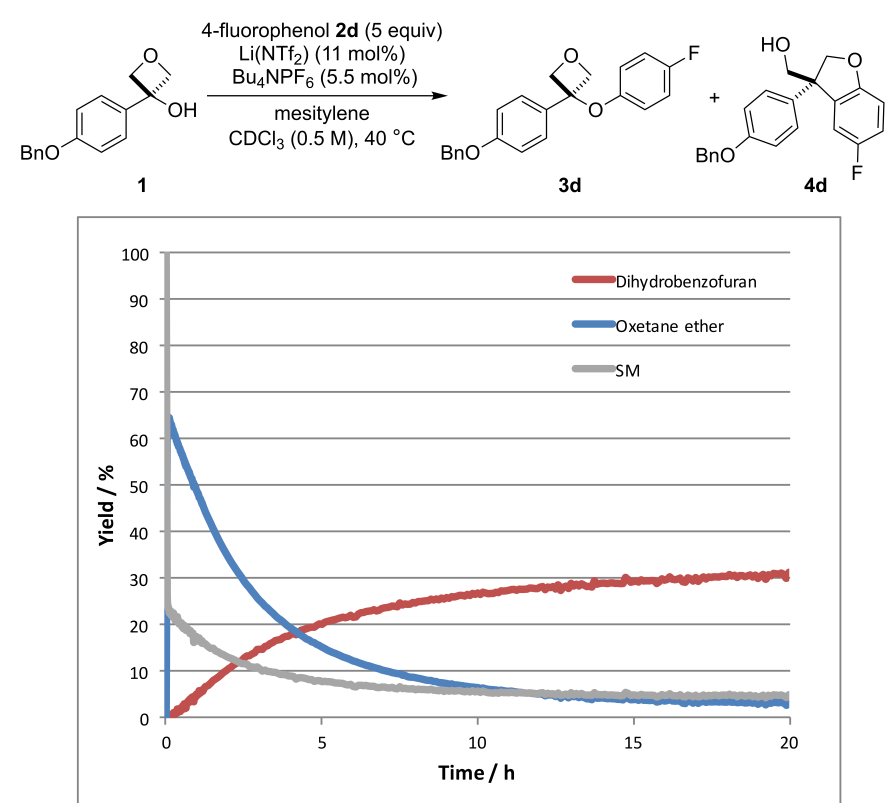

Figure 4. Reaction profile for the the reaction between 3-aryloxetan-3-ol and 4-fluorophenol 2d.

Next, a reaction profile for the reaction of oxetanol $\mathbf{1}$ and 4-methoxyphenol 2f was investigated using ${ }^{1} \mathrm{H} \quad \mathrm{NMR}$ spectroscopy (Figure 5). The oxetane ether product $3 \mathbf{f}$ was again formed very quickly in the reaction, reaching a maximum yield of $43 \%$ after just 11 minutes. The amount of oxetane ether $3 f$ then decreased until the reaction reached completion. Dimer product 5f, which is also a result of O-alkylation, formed reversibly in the reaction reaching a peak yield of $29 \%$ at approximately $2 \mathrm{~h}$ and then steadily decreased. The quantity of dihydrobenzofuran $\mathbf{4 f}$ increased throughout the reaction with a $77 \%$ yield on reaction completion. 

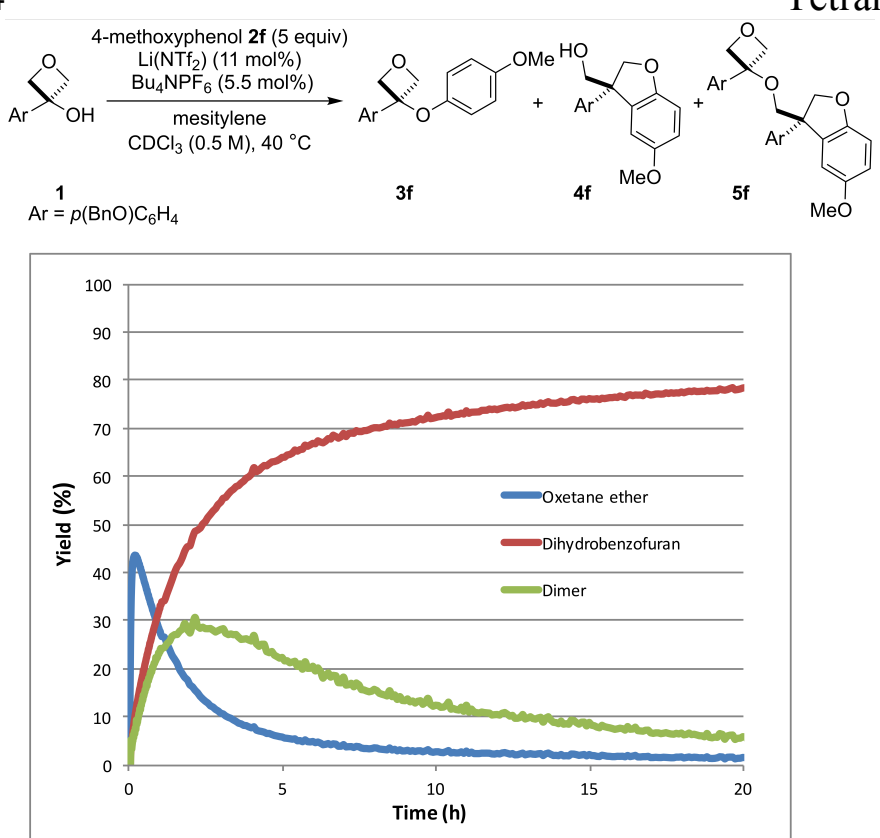

Figure 5. Reaction profile for the the reaction between oxetanol 1 and 4-methoxyphenol $\mathbf{2 f}$.

The reaction of oxetanol $\mathbf{1}$ and phenol itself (2g) was then examined, which allows for the formation of the diaryloxetane $\mathbf{6 g}$ (Figure 6). A similar trend was identified to the studies described above using phenols $\mathbf{2 d}$ and $\mathbf{2 f}$ in that oxetane ether $\mathbf{3 g}$ was formed reversibly as a kinetic product and the amount of the $\mathrm{C}$-alkylation products increased throughout the reaction. An approximately $3: 1$ ratio of $\mathbf{6 g}: \mathbf{4 g}$ was maintained throughout the reaction.
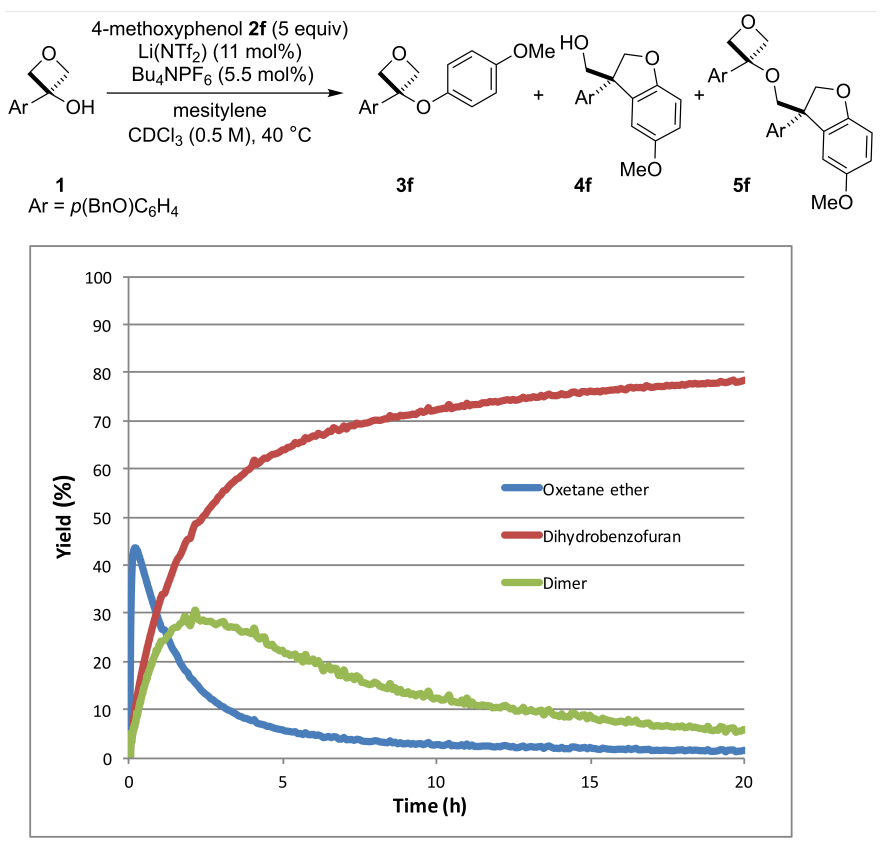

Figure 6 . Reaction profile for the the reaction between oxetanol 1 and phenol.

It is worth noting that in all cases where the dihydrobenzofuran product was formed, including when formed in high yield, the intermediate ortho-alkylated phenol was not observed. However, the nature of the product distribution is consistent with this being formed first, prior to oxetane ring opening. Interestingly, when the reaction between oxetanol $\mathbf{1}$ and ortho-methylphenol was explored in the same manner through ${ }^{1} \mathrm{H}$ NMR spectroscopy, no intermediate $O$-alkylation products were observed. The increased steric barrier of the ortho-methyl group may prevent $O$-alkylation and favor $C$-alkylation of the oxetanyl carbocation.

When the reaction was monitored using ${ }^{1} \mathrm{H}$ NMR spectroscopy without nucleophile present, a rapid degradation of starting material occurred. A small amount of an aldehyde side product was identified $(<6 \%$, scheme 3$)$, however, the majority of the material was unaccounted for. An insoluble precipitate was observed, indicating possible formation of a polymeric species under the reaction conditions resulting from ring opening of the oxetane, promoted by formation of the oxetane carbocation in the absence of a better nucleophile than the substrate itself.

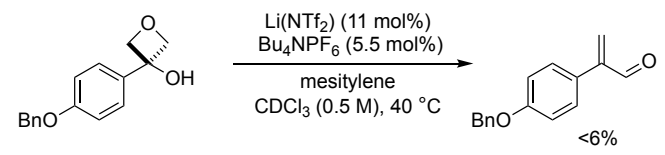

Scheme 3. Reaction in absence of nucleophile.

\subsection{Synthesis of oxetane ethers}

With careful control of the reaction time and set-up, the oxetane ether products were isolated in improved yield (Scheme 4). Reactions were set up in NMR tubes (on $0.25 \mathrm{mmol}$ scale, 0.5 $\mathrm{mL} \mathrm{CHCl}_{3}$ ) and reaction times were selected according to the time for the maximum yields seen in reaction profile studies (Section 2.2). The reaction with 4-fluorophenol gave a good isolated yield of $55 \%$ for oxetane ether $\mathbf{3 d}$ after just $10 \mathrm{~min}$ at $40{ }^{\circ} \mathrm{C}$. This compares well with the maximum observed yield of $64 \%$ observed by ${ }^{1} \mathrm{H}$ NMR. This could also be conducted on a larger $1.6 \mathrm{mmol}$ scale with only a small reduction in yield. Moderate yields were achieved with 4-methoxyphenol and phenol; however, these were also only slightly lower than the maximum potential yields identified in the corresponding NMR studies.
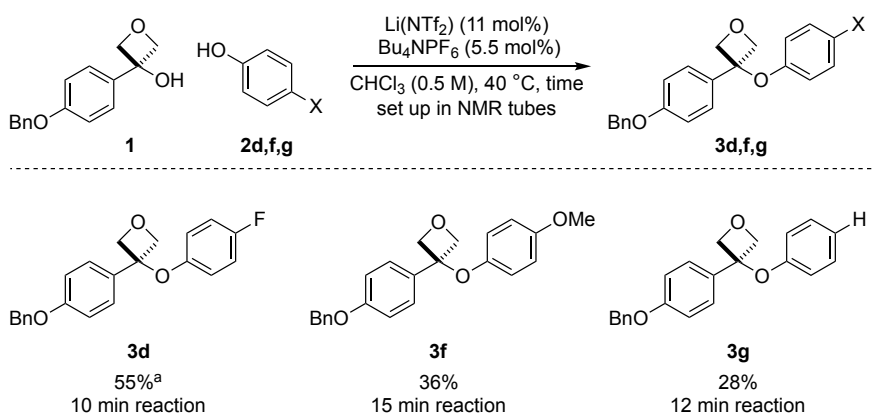

Scheme 4. Synthesis of oxetane ethers. Reactions conducted on a $0.25 \mathrm{mmol}$ scale. ${ }^{\mathrm{a}} 50 \%$ yield achieved on $1.6 \mathrm{mmol}$ scale.

To prove the reversibility of the formation of the oxetane ethers, ether 3d was resubmitted to the reaction conditions without further addition of nucleophile and also in the presence of 5 equiv of 4-methoxyphenol. Without additional added nucleophile, degradation was observed. However, in the presence of 4methoxyphenol (5 equiv) a 55\% yield of dihydrobenzofuran $\mathbf{4 f}$ was obtained. Indicating regeneration of a carbocationic intermediate which in the presence of an excess of the more reactive nucleophile can progress to the dihydrobenzofuran product. 


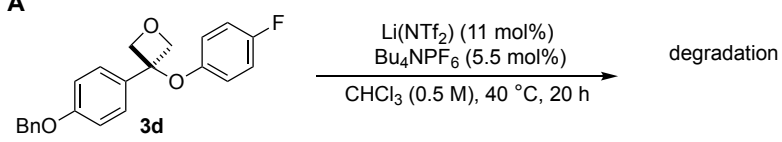

B

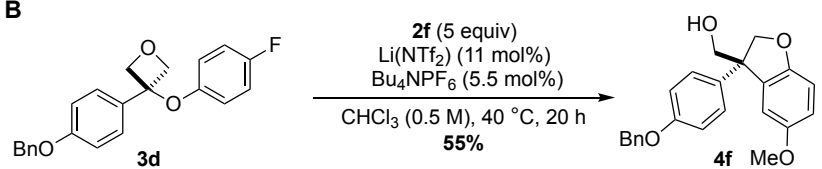

Scheme 5. Resubmission of oxetane ether to the Friedel-Crafts reaction conditions. A) Without additional nucleophile. B) With 5 equiv of 4-methoxyphenol added.

\subsection{Proposed Catalytic cycle}

With the information from these studies, a more complete catalytic cycle can be considered. An initial catalyst activation step is thought to involve solubilization of the lithium cation aided by the hexafluorophosphate additive though the precise nature of the catalytic species remains undefined. We propose that the catalytic species coordinates to the tertiary alcohol $\mathbf{1}$ to generate an oxetane carbocation. Addition of phenol 2 to the carbocation can occur either through the oxygen atom or aromatic carbon atoms. $O$-Alkylation occurs to give an oxetane ether 3, which is reversible. $C$-Alkylation at the para-position of the phenol occurs, where possible, to give the diaryloxetane product 6. Alternatively, $C$-alkylation at the ortho-position gives the dihydrobenzofuran $\mathbf{4}$ following rearomatisation and intramolecular oxetane ring opening. It is possible for the dihydrobenzofuran to react through the primary alcohol and add to a further equivalent of the oxetane carbocation to give a dimeric product 5 . The formation of this oxetane ether (5) is also reversible to regenerate the carbocation and eventually lead to the preferred diaryloxetane or dihydrobenzofuran products (6 or 4).

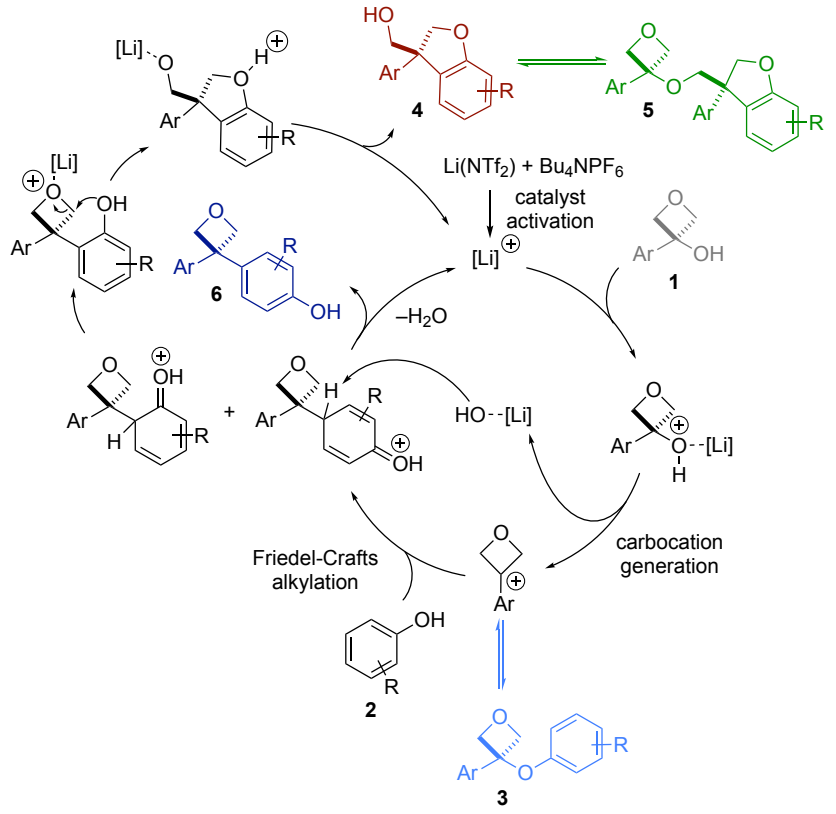

Figure 7. Proposed catalytic cycle with phenol as nucleophile.

\subsection{Identification of products by characteristic ${ }^{13} \mathrm{C} N \mathrm{NR}$ signals for oxetane $C-3$.}

Identification of the closely related isomeric products by ${ }^{1} \mathrm{H}$ NMR is made more complicated due to overlapping peaks in the aromatic region. However, across our studies to date, the ${ }^{13} \mathrm{C}$ NMR signal of the fully substituted oxetane C-3 atom has been diagnostic, enabling reliable preliminary characterization (Figure 8). For the oxetane ethers, with electron rich Ar groups, the oxetane quaternary carbon was consistently observed between 80.1 and $81.2 \mathrm{ppm}$ whereas for the diaryloxetane products this peak was seen at $46.0-51.0 \mathrm{ppm}$. This C-3 signal was also diagnostic for the dihydrobenzofuran and oxetanol starting material with ranges 55.1-57.1 ppm and 72.1-75.8 ppm respectively. This could be extended further to other oxetane derivatives, the ${ }^{13} \mathrm{C}$ peak in 3-sulfanyl-3-aryl-oxetanes was between 49.8-54.0 ppm, whereas the corresponding sulfones (68.2-69.3) and sulfoxides (67.2-67.4) are significantly different (though with fewer examples).

\begin{tabular}{|c|c|c|}
\hline & $\mathbf{X}=$ & $\begin{array}{c}\text { - Peak in }{ }^{13} \mathrm{C} \text { NMR } \\
\text { spectra/ ppm }\end{array}$ \\
\hline & OAr & $80.1-81.2$ \\
\hline & $\mathrm{Ar}$ & $46.0-51.0$ \\
\hline & $\mathrm{OH}$ & $72.1-75.8$ \\
\hline & & $49.8-54.0$ \\
\hline & $\mathrm{SO}_{2} \mathrm{R}$ & $68.2-69.3$ \\
\hline & SOR & $67.2-67.4$ \\
\hline
\end{tabular}

Figure 8. Characteristic oxetane quaternary carbon peaks for various 3,3-disubstituted oxetanes.

\section{Conclusion}

In conclusion, the reversible formation of oxetane ether intermediates in the Friedel-Crafts reaction between oxetanols and phenols has been studied. Changing the electronic nature of the para-substituent on phenol has shown an interesting relationship between electronics of the nucleophile with respect to the formation of the kinetic $O$-alkylation and thermodynamic $C$-alkylation products. For electron withdrawing 4-cyanophenol, the only product observed is the $O$-alkylation product whereas for halogens and electron rich substitutents formation of a mixture of $C$ - and $O$-alkylated products is observed at $1 \mathrm{~h}$ and exclusively the $C$-alkylation products after $20 \mathrm{~h}$. Reaction profiles generated using ${ }^{1} \mathrm{H}$ NMR spectroscopy reveal the reversible formation of the ether product, which is often consumed during the longer reaction times. These have enabled a more complete catalytic cycle to be proposed for the reaction. Furthermore, a number of the oxetane ether products have been isolated and are presented as novel potential bioisosteres for carboxylic esters.

\section{Experimental Section}

4.1. All nonaqueous reactions were run under an inert atmosphere (argon) with flame-dried glassware using standard techniques. $\mathrm{CHCl}_{3}(\geq 99.8 \%$, amylene stabilized $)$ was used directly from commercial sources without drying. Lithium triflimide (99.95\% trace metals basis) was purchased from Sigma Aldrich, used as supplied and stored in a desiccator between uses without further treatment. Flash column chromatography was performed using 230-400 mesh silica with the indicated solvent system according to standard techniques. Analytical thin-layer chromatography (TLC) was performed on precoated, glassbacked silica gel plates. Visualization of the developed chromatogram was performed by UV absorbance $(254 \mathrm{~nm})$, or PMA (phosphomolybdic acid). Infrared spectra ( $v_{\max }$, FTIR ATR) were recorded in reciprocal centimeters $\left(\mathrm{cm}^{-1}\right)$. Nuclear magnetic resonance spectra were recorded on $400 \mathrm{MHz}$ spectrometers. NMR tubes used for the reaction profile studies were purchased from Norell ${ }^{\circledR}$ (HP-507 grade). Chemical shifts for ${ }^{1} \mathrm{H}$ NMR spectra are recorded in parts per million from tetramethylsilane with the solvent resonance as the internal standard $\left(\mathrm{CDCl}_{3}, \delta=7.27 \mathrm{ppm}\right)$. Data is reported as follows: chemical shift [multiplicity $(\mathrm{s}=$ singlet, $\mathrm{d}=$ doublet, $\mathrm{t}=$ triplet, $\mathrm{m}$ $=$ multiplet and $\mathrm{br}=$ broad), coupling constant in $\mathrm{Hz}$, integration, assignment]. ${ }^{13} \mathrm{C}$ NMR spectra were recorded with complete 
proton decoupling. Chemical shifts are reported in parts per million from tetramethylsilane with the solvent resonance as the internal standard $\left({ }^{13} \mathrm{CDCl}_{3}: 77.0 \mathrm{ppm}\right) .{ }^{19} \mathrm{~F}$ NMR spectra were recorded with complete proton decoupling. Chemical shifts are reported in parts per million referenced to the standard monofluorobenzene: $-113.5 \mathrm{ppm}$. Chemical shifts are reported in parts per million. $\mathrm{J}$ values are reported in Hz. Assignments of ${ }^{1} \mathrm{H} /{ }^{13} \mathrm{C}$ spectra were made by the analysis of $\delta / \mathrm{J}$ values, and COSY, HSQC, and HMBC experiments as appropriate. Melting points were obtained using Optimelt MPA100 melting point apparatus and are uncorrected. For several of the dihydrobenzofurans the $\mathrm{O}-\mathrm{H}$ signal in the ${ }^{1} \mathrm{H}$ NMR was obscured by the water signal but confirmed by COSY spectra.

\subsection{3-[4-(Benzyloxy)phenyl]oxetan-3-ol (1) ${ }^{[13]}$}

$n$-BuLi (2.24 M in THF, $2.90 \mathrm{~mL}, 6.50 \mathrm{mmol}$ ) was added dropwise over $5 \mathrm{~min}$ to a solution of 1-(benzyloxy)-4bromobenzene $(1.71 \mathrm{~g}, 6.50 \mathrm{mmol})$ in THF $(20 \mathrm{~mL})$ at $-78{ }^{\circ} \mathrm{C}$. The reaction mixture was stirred at $-78{ }^{\circ} \mathrm{C}$ for a further $30 \mathrm{~min}$. Oxetan-3-one $(0.32 \mathrm{~mL}, 5.00 \mathrm{mmol})$ was added dropwise to the reaction mixture. Following a further $45 \mathrm{~min}$ at $-78^{\circ} \mathrm{C}$ the reaction mixture was warmed to $\mathrm{rt}$ then quenched with water $(20 \mathrm{~mL})$. The layers were separated and the aqueous portion extracted with diethylether $(3 \times 30 \mathrm{~mL})$. The organic extracts were combined, washed with brine, dried over $\mathrm{Na}_{2} \mathrm{SO}_{4}$, filtered and concentrated in vacuo. Purification by flash column chromatography (40\% EtOAc/hexane) afforded oxetanol $\mathbf{1}$ $(902 \mathrm{mg}, 70 \%)$ as a white solid. $\mathrm{R}_{f}=0.24$ (40\% EtOAc/hexane); $\mathrm{mp}=114-115{ }^{\circ} \mathrm{C}$; IR (film) $/ \mathrm{cm}-13394$ (br. OH), 2974, 2936, 2877, 1609, 1580, 1517, 1458, 1399, 1380, 1318, 1239, 1226, 1179, 1126, 1043, 1008, 958, 892, 843, 822, 756, 733, 701, 656; ${ }^{1} \mathrm{H}$ NMR $\left(400 \mathrm{MHz}, \mathrm{CDCl}_{3}\right) \delta 7.53-7.32(\mathrm{~m}, 7 \mathrm{H}, 7 \times \mathrm{Ar}-\mathrm{CH})$, 7.06-6.99 (m, 2 H, $2 \times \mathrm{Ar}-\mathrm{CH}), 5.10\left(\mathrm{~s}, 2 \mathrm{H}, \mathrm{Ph}-\mathrm{CH}_{2}\right), 4.93$ (d, $J$ $=6.9 \mathrm{~Hz}, 2 \mathrm{H}, \mathrm{CH} \mathrm{HOCHH}), 4.90(\mathrm{~d}, J=6.9 \mathrm{~Hz}, 2 \mathrm{H}$, $\mathrm{CH} H \mathrm{OCH} H), 2.42(\mathrm{~s}, 1 \mathrm{H}, \mathrm{OH}) ;{ }^{13} \mathrm{C} \mathrm{NMR}\left(101 \mathrm{MHz}, \mathrm{CDCl}_{3}\right)$ $\delta 158.4\left(\mathrm{Ar}-\mathrm{C}_{\mathrm{q}} \mathrm{-O}\right), 136.8\left(\mathrm{Ph}-\mathrm{C}_{\mathrm{q}}\right), 134.7\left(\mathrm{Ar}-\mathrm{C}_{q}-\mathrm{C}_{\mathrm{q}}\right), 128.6(2 \times$ Ar-CH), 128.0 (Ar-CH), $127.4(2 \times$ Ar-CH), $125.9(2 \times$ Ar-CH $)$, $115.0(2 \times \mathrm{Ar}-\mathrm{CH}), 85.5\left(\mathrm{CH}_{2} \mathrm{OCH}_{2}\right), 75.7\left(\mathrm{C}_{\mathrm{q}}\right), 70.1\left(\mathrm{PhCH}_{2}\right)$; HRMS (ESI-TOF) $\mathrm{m} / \mathrm{z}$ calcd for $\mathrm{C}_{16} \mathrm{H}_{15} \mathrm{O}_{2}{ }^{+}[\mathrm{M}-\mathrm{OH}]^{+}:$239.1072, Found: 239.1068. The observed spectroscopic data for this compound was consistent with that previously reported. ${ }^{[18]}$

\subsection{General Procedure A:}

Lithium bis(trifluoromethanesulfonimide) $(7.9 \mathrm{mg}, 0.0275$ $\mathrm{mmol})$ and tetrabutylammonium hexafluorophosphate $(5.3 \mathrm{mg}$, $0.0138 \mathrm{mmol})$ were added to a solution of oxetanol $1(64 \mathrm{mg}$, $0.25 \mathrm{mmol})$ and phenol 2a-2f $(1.25 \mathrm{mmol})$ in chloroform $(0.5 \mathrm{~mL})$. The reaction mixture was stirred at $40{ }^{\circ} \mathrm{C}$ for either $1 \mathrm{~h}$ or $20 \mathrm{~h}$ then quenched with sat. aq. $\mathrm{NaHCO}_{3}$. The layers were separated and the aqueous portion extracted with dichloromethane $(3 \times 5 \mathrm{~mL})$. The organic extracts were combined, washed with aq. $\mathrm{NaOH}(1 \mathrm{M}, 15 \mathrm{~mL})$ then dried over $\mathrm{Na}_{2} \mathrm{SO}_{4}$, filtered and concentrated in vacuo. Purification by flash column chromatography afforded $C$-alkylated (4) and/or $O$-alkylated product(s) (3 and/or 5).

\subsubsection{4-(\{3-[4-(Benzyloxy)phenyl]oxetan-3-} yl\}oxy)benzonitrile (3a)

Prepared according to General Procedure A described above, using 4-cyanophenol (149 mg, $1.25 \mathrm{mmol})$ and a reaction time of
$1 \mathrm{~h}$. Purification by flash chromatography (30\% EtOAc/hexane) afforded oxetane ether 3a (14 mg, 16\%) as a white solid.

After $20 \mathrm{~h}$ the same procedure afforded oxetane ether 3a (21 $\mathrm{mg}, 23 \%$ ) as a white solid.

Oxetane ether 3a: $\mathrm{R}_{f}=0.37$ (30\% EtOAc/hexane); $\mathrm{mp}=164$ $166^{\circ} \mathrm{C}$; IR (film) $/ \mathrm{cm}^{-1} 2948,2879,2226$ (CN stretch), 1605, $1582,1505,1455,1297,1247,1172,1112,1010,987,900,832$, 737, 698; ${ }^{1} \mathrm{H} \mathrm{NMR}\left(400 \mathrm{MHz}, \mathrm{CDCl}_{3}\right) \delta 7.52-7.35(\mathrm{~m}, 9 \mathrm{H}, 9 \times$ Ar-CH), 7.05-7.00 (m, 2 H, $2 \times$ Ar-CH), 6.63-6.59 (m, 2 H, $2 \times$ Ar-CH), $5.09(\mathrm{~d}, J=7.0 \mathrm{~Hz}, 2 \mathrm{H}, \mathrm{CH} \mathrm{HOCH}), 5.08(\mathrm{~s}, 2 \mathrm{H}$, $\left.\mathrm{PhCH}_{2}\right), 4.96(\mathrm{~d}, J=7.0 \mathrm{~Hz}, 2 \mathrm{H}, \mathrm{CH} H \mathrm{OCH} H) ;{ }^{13} \mathrm{C}$ NMR $(101$ $\left.\mathrm{MHz}, \mathrm{CDCl}_{3}\right) \delta 158.7\left(\mathrm{Ar}-\mathrm{C}_{\mathrm{q}}-\mathrm{O}\right), 158.4\left(\mathrm{Ar}-\mathrm{C}_{\mathrm{q}}-\mathrm{O}\right), 136.6\left(\mathrm{Ph}-C_{q^{-}}\right.$ $\left.\mathrm{CH}_{2}\right), 133.9(2 \times \mathrm{Ar}-\mathrm{CH}), 130.8\left(\mathrm{Ar}-\mathrm{C}_{q}-\mathrm{C}_{\mathrm{q}}\right) 128.7(2 \times \mathrm{Ar}-\mathrm{CH})$, $128.2(\mathrm{Ar}-\mathrm{CH}), 127.5(2 \times \mathrm{Ar}-\mathrm{CH}), 125.9(2 \times \mathrm{Ar}-\mathrm{CH}), 118.9$ $(\mathrm{CN}), 117.1(2 \times \mathrm{Ar}-\mathrm{CH}), 115.4(2 \times \mathrm{Ar}-\mathrm{CH}), 104.8\left(\mathrm{Ar}-C_{q}-\mathrm{CN}\right)$, $82.5\left(\mathrm{CH}_{2} \mathrm{OCH}_{2}\right), 81.2\left(\mathrm{C}_{\mathrm{q}}\right), 70.1\left(\mathrm{Ph}-\mathrm{CH}_{2}\right) ; \mathrm{FTMS}(+\mathrm{pNSI}) \mathrm{m} / \mathrm{z}$ calcd for $\mathrm{C}_{23} \mathrm{H}_{19} \mathrm{NO}_{3} \mathrm{Na}^{+}[\mathrm{M}+\mathrm{Na}]^{+}: 380.1257$, Found: 380.1256 .

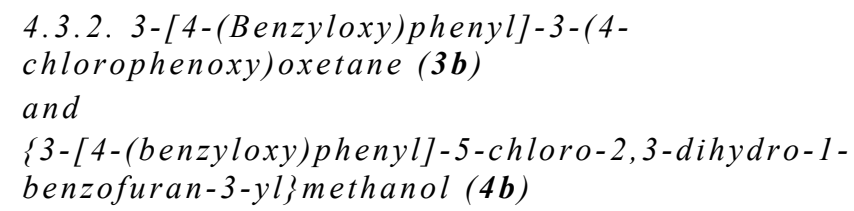

Prepared according to General Procedure A described above, using 4-chlorophenol (161 $\mathrm{mg}, 1.25 \mathrm{mmol})$ and a reaction time of $1 \mathrm{~h}$. Purification by flash chromatography (20\% EtOAc/hexane) afforded afforded oxetane ether $\mathbf{3 b}(24 \mathrm{mg}, 26 \%)$ as a white solid followed by dihydrobenzofuran $\mathbf{4 b}(8 \mathrm{mg}, 9 \%)$ as a colorless gum.

After $20 \mathrm{~h}$ the same procedure afforded dihydrobenzofuran $\mathbf{4 b}$ (26 mg, 28\%).

Oxetane ether 3b: $\mathrm{R}_{f}=0.47$ (20\% EtOAc/hexane); $\mathrm{mp}=120$ $125^{\circ} \mathrm{C}$; IR (film) $/ \mathrm{cm}^{-1}$ 2950, 2876, 1610, 1582, 1487, 1455, $1382,1283,1283,1235,1179,1112,1091,1008,987,899,824$, 736, 697, 667; ${ }^{1} \mathrm{H}$ NMR $\left(400 \mathrm{MHz}, \mathrm{CDCl}_{3}\right) \delta 7.49-7.34(\mathrm{~m}, 7 \mathrm{H}$, $7 \times \mathrm{Ar}-\mathrm{CH}), 7.16-7.11(\mathrm{~m}, 2 \mathrm{H}, 2 \times \mathrm{Ar}-\mathrm{CH}), 7.03-6.98(\mathrm{~m}, 2 \mathrm{H}$, $2 \times \mathrm{Ar}-\mathrm{CH}), 6.49-6.44(\mathrm{~m}, 2 \mathrm{H}, 2 \times \mathrm{Ar}-\mathrm{CH}), 5.08(\mathrm{~d}, J=6.9 \mathrm{~Hz}$, $2 \mathrm{H}, \mathrm{CH} \mathrm{HOCHH}), 5.07\left(\mathrm{~s}, 2 \mathrm{H}, \mathrm{PhCH}_{2}\right), 4.91(\mathrm{~d}, J=6.9 \mathrm{~Hz}, 2 \mathrm{H}$, $\mathrm{CH} H \mathrm{OCH} H) ;{ }^{13} \mathrm{C}$ NMR $\left(101 \mathrm{MHz}, \mathrm{CDCl}_{3}\right) \delta 158.5\left(\mathrm{Ar}-C_{q^{-}}\right.$ $\mathrm{OBn}), 153.4\left(\mathrm{Ar}_{(p-\mathrm{Cl})}-C_{q}-\mathrm{O}\right), 136.7\left(\mathrm{Ph}-C_{q}-\mathrm{CH}_{2}\right), 131.7\left(\mathrm{Ar}-C_{q^{-}}-\right.$ $\left.\mathrm{C}_{\mathrm{q}}\right), 129.3(2 \times \mathrm{Ar}-\mathrm{CH}), 128.6(2 \times \mathrm{Ar}-\mathrm{CH}), 128.0(\mathrm{Ar}-\mathrm{CH})$, $127.5(2 \times \mathrm{Ar}-\mathrm{CH}), 126.10\left(\mathrm{Ar}-\mathrm{C}_{q}-\mathrm{Cl}\right), 126.06(2 \times \mathrm{Ar}-\mathrm{CH})$, $117.6(2 \times \mathrm{Ar}-\mathrm{CH}), 115.2(2 \times \mathrm{Ar}-\mathrm{CH}), 82.7\left(\mathrm{CH}_{2} \mathrm{OCH}_{2}\right), 80.5$ $\left(\mathrm{C}_{\mathrm{q}}\right), \quad 70.1 \quad\left(\mathrm{Ph}-\mathrm{CH}_{2}\right)$; HRMS (ESI-TOF) $\mathrm{m} / \mathrm{z}$ calcd for $\mathrm{C}_{22} \mathrm{H}_{20} \mathrm{O}_{3} \mathrm{Cl}^{+}[\mathrm{M}+\mathrm{H}]^{+}:$367.1101, Found: 367.1096.

Dihydrobenzofuran 4b: $\mathrm{R}_{f}=0.18(20 \%$ EtOAc/hexane); IR (film) $/ \mathrm{cm}^{-1}$ 2880, 1609, 1512, 1479, 1381, 1297, 1244, 1183, 1025, 975, 827, 736, 696; ${ }^{1} \mathrm{H}$ NMR $\left(400 \mathrm{MHz}, \mathrm{CDCl}_{3}\right) \delta 7.46-$ $7.33(\mathrm{~m}, 5 \mathrm{H}, 5 \times \mathrm{Ar}-\mathrm{CH}), 7.25-7.15(\mathrm{~m}, 4 \mathrm{H}, 4 \times \mathrm{Ar}-\mathrm{CH}), 7.00$ 6.95 (m, 2 H, $2 \times$ Ar-CH), 6.85-6.79 (m, $1 \mathrm{H}, \mathrm{Ar}-\mathrm{CH}), 5.07$ (s, 2 $\left.\mathrm{H}, \mathrm{Ph}-\mathrm{CH}_{2}\right), 4.77(\mathrm{dd}, J=9.1 \mathrm{~Hz}, 1 \mathrm{H}, \mathrm{OCHHC}$ ), 4.59 (dd, $J=$ $9.0 \mathrm{~Hz}, 1 \mathrm{H}, \mathrm{OCH}_{H C}$ ), $4.11(\mathrm{dd}, J=11.2,4.6 \mathrm{~Hz}, 1 \mathrm{H}$, $\mathrm{C} H \mathrm{HOH}), 4.03(\mathrm{dd}, J=11.2,7.1 \mathrm{~Hz}, 1 \mathrm{H}, \mathrm{CH} H \mathrm{OH}) ;{ }^{13} \mathrm{C} \mathrm{NMR}$ $\left(101 \mathrm{MHz}, \mathrm{CDCl}_{3}\right) \delta 159.4\left(\mathrm{Ar}-\mathrm{C}_{\mathrm{q}}-\mathrm{O}\right), 157.9\left(\mathrm{Ar}-C_{q}-\mathrm{O}\right), 136.8$ $\left(\mathrm{Ph}-C_{q}-\mathrm{CH}_{2}\right), 134.1\left(\mathrm{Ar}-C_{q}-\mathrm{C}_{\mathrm{q}}\right), 132.4\left(\mathrm{Ar}-C_{q}-\mathrm{C}_{\mathrm{q}}\right), 129.1(\mathrm{Ar}-$ $\mathrm{CH}), 128.6(2 \times \mathrm{Ar}-\mathrm{CH}), 128.1(\mathrm{Ar}-\mathrm{CH}), 128.0(2 \times \mathrm{Ar}-\mathrm{CH})$, $127.5(2 \times \mathrm{Ar}-\mathrm{CH}), 125.5\left(\mathrm{Ar}-\mathrm{C}_{q}-\mathrm{Cl}\right), 125.4(\mathrm{Ar}-\mathrm{CH}), 115.2(2 \times$ Ar-CH), $111.2(\mathrm{Ar}-\mathrm{CH}), 81.9\left(\mathrm{OCH}_{2} \mathrm{C}_{\mathrm{q}}\right), 70.1\left(\mathrm{Ph}-\mathrm{CH}_{2}\right), 67.5$ $\left(\mathrm{CH}_{2} \mathrm{OH}\right), 55.9\left(\mathrm{C}_{\mathrm{q}}\right)$; FTMS (+pNSI) $\mathrm{m} / z$ calcd for $\mathrm{C}_{22} \mathrm{H}_{23} \mathrm{ClO}_{3} \mathrm{~N}^{+}$ $\left[\mathrm{M}+\mathrm{NH}_{4}\right]^{+}$: 384.1361, Found: 384.1356. The observed 
spectroscopic data for this compound was consistent with that previously reported. ${ }^{[13]}$

\author{
4.3.3. 3-[4-(Benzyloxy)phenyl]-3-(4- \\ iodophenoxy)oxetane (3c) \\ and \\ \{3-[4-(benzyloxy)phenyl]-5-iodo-2,3-dihydro-1- \\ benzofuran-3-yl\}methanol (4c)
}

Prepared according to General Procedure A described above, using 4-iodophenol $(275 \mathrm{mg}, 1.25 \mathrm{mmol})$ and a reaction time of $1 \mathrm{~h}$. Purification by flash chromatography $(20 \%$ EtOAc/hexane) afforded afforded oxetane ether $3 \mathbf{c}(10 \mathrm{mg}, 8 \%)$ as a white solid followed by dihydrobenzofuran $\mathbf{4 c}(32 \mathrm{mg}, 28 \%)$ as a colorless gum.

After $20 \mathrm{~h}$ the same procedure afforded dihydrobenzofuran $\mathbf{4 c}$ (27 mg, 25\%).

Oxetane ether 3c: $\mathrm{R}_{f}=0.54(20 \%$ EtOAc/hexane); $\mathrm{mp}=112$ $114{ }^{\circ} \mathrm{C}$; IR (film) $/ \mathrm{cm}^{-1}$ 2872, 1612, 1578, 1511, 1482, 1456, $1385,1301,1279,1234,1176,1113,1058,1025,1001,986,958$, $899,831,813,745,698,679 ;{ }^{1} \mathrm{H}$ NMR $\left(400 \mathrm{MHz}, \mathrm{CDCl}_{3}\right)$ $\delta$ 7.48-7.32 (m, $9 \mathrm{H}, 9 \times \mathrm{Ar}-\mathrm{CH}), 7.01-6.97(\mathrm{~m}, 2 \mathrm{H}, 2 \times \mathrm{Ar}-$ $\mathrm{CH}), 6.35-6.29$ (m, $2 \mathrm{H}, 2 \times \mathrm{Ar}-\mathrm{CH}), 5.07(\mathrm{~d}, J=7.4 \mathrm{~Hz}, 2 \mathrm{H}$, $\mathrm{C} H \mathrm{HOCH}), 5.06\left(\mathrm{~s}, 2 \mathrm{H}, \mathrm{PhCH}_{2}\right), 4.90(\mathrm{~d}, J=7.4 \mathrm{~Hz}, 2 \mathrm{H}$, $\mathrm{CH} H \mathrm{OCH} H) ;{ }^{13} \mathrm{C}$ NMR $\left(101 \mathrm{MHz}, \mathrm{CDCl}_{3}\right) \delta 158.6\left(\mathrm{Ar}-\mathrm{C}_{q^{-}}\right.$ $\left.\mathrm{OCH}_{2}\right), 154.8\left(\mathrm{Ar}-C_{q^{-}}-\mathrm{OC}_{\mathrm{q}}\right), 138.3(2 \times \mathrm{Ar}-\mathrm{CH}), 136.7\left(\mathrm{Ar}-C_{q^{-}}\right.$ $\left.\mathrm{C}_{\mathrm{q}}\right), 131.6\left(\mathrm{Ph}-\mathrm{C}_{\mathrm{q}}-\mathrm{CH}_{2}\right), 128.6(2 \times \mathrm{Ar}-\mathrm{CH}), 128.1(\mathrm{Ar}-\mathrm{CH})$, $127.5(2 \times$ Ar-CH $), 126.0(2 \times$ Ar-CH $), 118.7(2 \times$ Ar-CH $), 115.2$ $(2 \times \mathrm{Ar}-\mathrm{CH}), 83.5\left(\mathrm{Ar}-\mathrm{C}_{\mathrm{q}}-\mathrm{I}\right), 82.7\left(\mathrm{CH}_{2} \mathrm{OCH}_{2}\right), 80.5\left(\mathrm{C}_{\mathrm{q}}\right), 70.1$ $\left(\mathrm{Ph}-\mathrm{CH}_{2}\right)$; FTMS $(+\mathrm{pNSI}) \mathrm{m} / z$ calcd for $\mathrm{C}_{22} \mathrm{H}_{23} \mathrm{O}_{3} \mathrm{NI}^{+}\left[\mathrm{M}+\mathrm{NH}_{4}\right]^{+}$: 476.0717, Found: 476.0714.

Dihydrobenzofuran 4c: $\mathrm{R}_{f}=0.26(20 \%$ EtOAc/hexane); IR (film) $/ \mathrm{cm}^{-1} 3429$ (br, OH), 3032, 2880, 1609, 1582, 1476, 1456, 1382, 1293, 1238, 1183, 1130, 1025, 971, 909, 827, 811, 734, 697, 669; ${ }^{1} \mathrm{H}$ NMR $\left(400 \mathrm{MHz}, \mathrm{CDCl}_{3}\right) \delta 7.53-7.33(\mathrm{~m}, 7 \mathrm{H}, 7 \times$ Ar-CH), 7.24-7.18 (m, 2 H, $2 \times$ Ar-CH), 7.00-6.96 (m, 2 H, $2 \times$ Ar- $\mathrm{CH}), 6.71-6.67$ (m, 1 H, Ar-CH), 5.07 (s, $\left.2 \mathrm{H}, \mathrm{PhCH}_{2}\right), 4.75$ $\left(\mathrm{d}, J=9.0 \mathrm{~Hz}, 1 \mathrm{H}, \mathrm{OCHHC}_{\mathrm{q}}\right), 4.57(\mathrm{~d}, J=9.0 \mathrm{~Hz}, 1 \mathrm{H}$, $\mathrm{OCH}_{H \mathrm{q}}$ ), 4.09 (d, $\left.J=11.2 \mathrm{~Hz}, 1 \mathrm{H}, \mathrm{CHHOH}\right), 4.02(\mathrm{~d}, J=11.2$ $\mathrm{Hz}, 1 \mathrm{H}, \mathrm{CH} H \mathrm{OH}) ;{ }^{13} \mathrm{C}$ NMR $\left(101 \mathrm{MHz}, \mathrm{CDCl}_{3}\right) \delta 160.6$ $\left(\mathrm{Ar}_{(\mathrm{DHBF})}-\mathrm{C}_{q}-\mathrm{OCH}_{2}\right), 157.9\left(\mathrm{Ar}-\mathrm{C}_{\mathrm{q}}-\mathrm{OBn}\right), 137.9(\mathrm{Ar}-\mathrm{CH}), 136.8$ $\left(\mathrm{Ph}-\mathrm{C}_{\mathrm{q}}\right), 134.1\left(\mathrm{Ar}-\mathrm{C}_{q}-\mathrm{C}_{\mathrm{q}}\right), 133.9(\mathrm{Ar}-\mathrm{CH}), 133.6\left(\mathrm{Ar}-\mathrm{C}_{q}-\mathrm{C}_{\mathrm{q}}\right)$, $128.6(2 \times \mathrm{Ar}-\mathrm{CH}), 128.01(\mathrm{Ar}-\mathrm{CH}), 127.97(2 \times \mathrm{Ar}-\mathrm{CH}), 127.4$ $(2 \times \mathrm{Ar}-\mathrm{CH}), 115.2(2 \times \mathrm{Ar}-\mathrm{CH}), 112.5(\mathrm{Ar}-\mathrm{CH}), 82.1\left(\mathrm{Ar}-\mathrm{C}_{\mathrm{q}}-\mathrm{I}\right)$, $81.7\left(\mathrm{OCH}_{2} \mathrm{C}_{\mathrm{q}}\right), 70.0\left(\mathrm{Ph}_{-} \mathrm{CH}_{2}\right), 67.5\left(\mathrm{CH}_{2} \mathrm{OH}\right), 55.6\left(\mathrm{C}_{\mathrm{q}}\right)$; FTMS (+pNSI) $m / z$ calcd for $\mathrm{C}_{22} \mathrm{H}_{23} \mathrm{O}_{3} \mathrm{NI}^{+}\left[\mathrm{M}+\mathrm{NH}_{4}\right]^{+}: 476.0717$, Found: 476.0709. The observed spectroscopic data for this compound was consistent with that previously reported. ${ }^{[13]}$

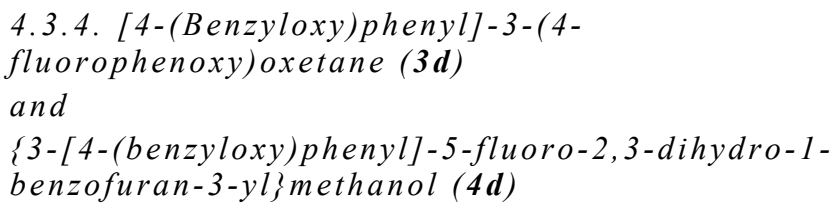

Prepared according to General Procedure A described above, using 4-fluorophenol $(140 \mathrm{mg}, 1.25 \mathrm{mmol})$ and a reaction time of $1 \mathrm{~h}$. Purification by flash chromatography (20\% EtOAc/hexane) afforded oxetane ether $\mathbf{3 d}(28 \mathrm{mg}, 32 \%)$ as a white solid followed by dihydrobenzofuran $\mathbf{4 d}(5 \mathrm{mg}, 6 \%)$ as a white solid.
After $20 \mathrm{~h}$ the same procedure afforded dihydrobenzofuran $4 \mathrm{~d}$ (25 mg, 29\%).

Oxetane ether 3d: $\mathrm{R}_{f}=0.37$ (20\% EtOAc/hexane); $\mathrm{mp}=100$ $102{ }^{\circ} \mathrm{C}$; IR (film) $/ \mathrm{cm}^{-1} 2950,2876,1609,1500,1455,1382$, 1294, 1241, 1207, 1179, 1112, 1098, 1009, 986, 900, 826, 744, 697; ${ }^{1} \mathrm{H}$ NMR $\left(400 \mathrm{MHz}, \mathrm{CDCl}_{3}\right) \delta$ 7.51-7.32 (m, $7 \mathrm{H}, 7 \times \mathrm{Ar}-$ $\mathrm{CH}), 7.03-6.97$ (m, $2 \mathrm{H}, 2 \times \mathrm{Ar}-\mathrm{CH}), 6.91-6.81$ (m, $2 \mathrm{H}, 2 \times \mathrm{Ar}-$ $\mathrm{CH}), 6.51-6.44(\mathrm{~m}, 2 \mathrm{H}, 2 \times \mathrm{Ar}-\mathrm{CH}), 5.08(\mathrm{~d}, J=6.9 \mathrm{~Hz}, 2 \mathrm{H}$, $\mathrm{C} H \mathrm{HOCHH}), 5.07\left(\mathrm{~s}, 2 \mathrm{H}, \mathrm{PhCH}_{2}\right), 4.91(\mathrm{~d}, J=6.9 \mathrm{~Hz}, 2 \mathrm{H}$, $\mathrm{CH} H \mathrm{OCH} H) ;{ }^{13} \mathrm{C}$ NMR $\left(101 \mathrm{MHz}, \mathrm{CDCl}_{3}\right) \delta 158.5\left(\mathrm{Ar}-\mathrm{C}_{q^{-}}\right.$ $\mathrm{OBn}), 157.3\left(\mathrm{~d}, J_{C-F}=240.5 \mathrm{~Hz}, \mathrm{Ar}-C_{q}-\mathrm{F}\right), 150.9\left(\mathrm{Ar}-C_{q}-\mathrm{OC}_{\mathrm{q}}\right)$, $136.7\left(\mathrm{Ph}-C_{q}-\mathrm{CH}_{2}\right), 132.0\left(\mathrm{Ar}-C_{q}-\mathrm{C}_{\mathrm{q}}\right), 128.6(2 \times \mathrm{Ar}-\mathrm{CH}), 128.1$ $(\mathrm{Ar}-\mathrm{CH}), 127.5(2 \times \mathrm{Ar}-\mathrm{CH}), 126.1(2 \times \mathrm{Ar}-\mathrm{CH}), 117.3\left(\mathrm{~d}, J_{\mathrm{C}-F}=\right.$ $8.3 \mathrm{~Hz}, 2 \times \mathrm{Ar}-\mathrm{CH}), 115.8\left(\mathrm{~d}, J_{C-F}=23.1 \mathrm{~Hz}, 2 \times \mathrm{Ar}-\mathrm{CH}\right), 115.1$ $(2 \times \mathrm{Ar}-\mathrm{CH}), 82.6\left(\mathrm{CH}_{2} \mathrm{OCH}_{2}\right), 80.5\left(\mathrm{C}_{\mathrm{q}}\right), 70.1\left(\mathrm{Ph}-\mathrm{CH}_{2}\right) ;{ }^{19} \mathrm{~F}$ NMR $\left(377 \mathrm{MHz}, \mathrm{CDCl}_{3}\right) \delta-123.3$; FTMS (+ pNSI) $\mathrm{m} / z$ calcd for $\mathrm{C}_{22} \mathrm{H}_{23} \mathrm{O}_{3} \mathrm{FNa}^{+}[\mathrm{M}+\mathrm{Na}]^{+}: 373.1210$, Found: 373.1216.

Dihydrobenzofuran 4d: $\mathrm{R}_{f}=0.11(20 \%$ EtOAc/hexane $) ; \mathrm{mp}=$ 92-94 ${ }^{\circ} \mathrm{C}$; IR (film) $/ \mathrm{cm}^{-1} 3439$ (br. OH), 2875, 1609, 1501, 1511, 1485, 1382, 1296, 1246, 1183, 1025, 978, 863, 828, 813, 775, 738, 697; ${ }^{1} \mathrm{H}$ NMR $\left(400 \mathrm{MHz}, \mathrm{CDCl}_{3}\right) \delta 7.47-7.33(\mathrm{~m}, 5 \mathrm{H}, 5 \times$ Ar-CH), 7.25-7.21 (m, 2 H, $2 \times$ Ar-CH), 7.00-6.89 (m, 4 H, $4 \times$ Ar-CH), 6.83-6.78 (m, $1 \mathrm{H}, 1 \times \mathrm{Ar}-\mathrm{CH}), 5.07\left(\mathrm{~s}, 2 \mathrm{H}, \mathrm{PhCH}_{2}\right)$, $4.76\left(\mathrm{~d}, J=9.0 \mathrm{~Hz}, 1 \mathrm{H}, \mathrm{OC} H \mathrm{HC}_{\mathrm{q}}\right), 4.58(\mathrm{~d}, J=9.0 \mathrm{~Hz}, 1 \mathrm{H}$, $\left.\mathrm{OCH} \mathrm{C}_{\mathrm{q}}\right), 4.12(\mathrm{dd}, J=11.1,4.9 \mathrm{~Hz}, 1 \mathrm{H}, \mathrm{CH} \mathrm{HOH}), 4.03(\mathrm{dd}, J$ $=11.1,7.6 \mathrm{~Hz}, 1 \mathrm{H}, \mathrm{CH} H \mathrm{OH}) ;{ }^{13} \mathrm{C} \mathrm{NMR}\left(101 \mathrm{MHz}, \mathrm{CDCl}_{3}\right) \delta$ $157.8\left(\mathrm{Ar}-\mathrm{C}_{\mathrm{q}}-\mathrm{OBn}\right), 157.5\left(\mathrm{~d}, J_{C-F}=240.5 \mathrm{~Hz}, \mathrm{Ar}-C_{q}-\mathrm{F}\right), 152.8$ $\left(\mathrm{Ar}_{(\mathrm{DHBF})}-C_{q}-\mathrm{OCH}_{2}\right), 136.8\left(\mathrm{Ph}-\mathrm{C}_{\mathrm{q}}\right), 134.1\left(\mathrm{Ar}_{(p-\mathrm{OBn})}-C_{q}-\mathrm{C}_{\mathrm{q}}\right), 129.3$ $\left(\mathrm{Ar}_{(\mathrm{DHBF})}-\mathrm{C}_{q}-\mathrm{C}_{\mathrm{q}}\right), 128.6(2 \times \mathrm{Ar}-\mathrm{CH}), 128.03(2 \times \mathrm{Ar}-\mathrm{CH}), 127.98$ $(\mathrm{Ar}-\mathrm{CH}), 127.4(2 \times \mathrm{Ar}-\mathrm{CH}), 115.5\left(\mathrm{~d}, J_{C-F}=23.9 \mathrm{~Hz}, \mathrm{Ar}-\mathrm{CH}\right)$, $115.2(2 \times \mathrm{Ar}-\mathrm{CH}), 112.3\left(\mathrm{~d}, J_{C-F}=25.4 \mathrm{~Hz}, \mathrm{Ar}-\mathrm{CH}\right), 110.4(\mathrm{~d}$, $\left.J_{C-F}=8.2 \mathrm{~Hz}, \mathrm{Ar}-\mathrm{CH}\right), 81.8\left(\mathrm{OCH}_{2} \mathrm{C}_{\mathrm{q}}\right), 70.0\left(\mathrm{PhCH}_{2}\right), 67.5$ $\left(\mathrm{CH}_{2} \mathrm{OH}\right), 55.9\left(\mathrm{C}_{\mathrm{q}}\right) ;{ }^{19} \mathrm{~F}$ NMR $\left(377 \mathrm{MHz}, \mathrm{CDCl}_{3}\right) \delta-123.5$; FTMS (+pNSI) $m / z$ calcd for $\mathrm{C}_{22} \mathrm{H}_{23} \mathrm{O}_{3} \mathrm{NF}^{+}\left[\mathrm{M}+\mathrm{NH}_{4}\right]^{+}: 368.1656$, Found: 368.1659. The observed spectroscopic data for this compound was consistent with that previously reported. ${ }^{[13]}$

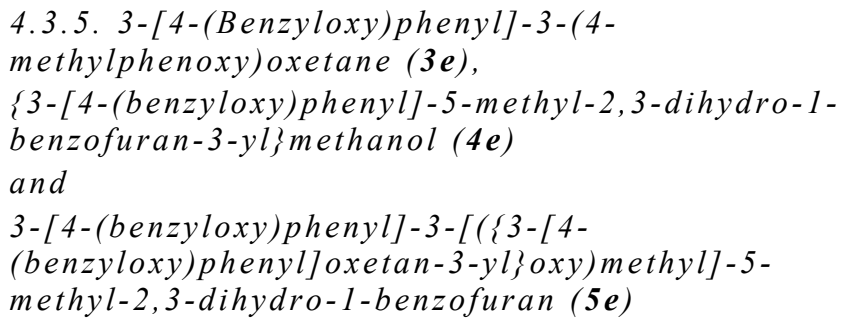

Prepared according to General Procedure A described above, using 4-methylphenol (135 mg, $1.25 \mathrm{mmol})$ and a reaction time of $1 \mathrm{~h}$. Purification by flash chromatography $(30 \%$ EtOAc/hexane) afforded oxetane ether $3 \mathbf{e}(6 \mathrm{mg}, 3 \%)$ as a white solid followed dimer $\mathbf{5 e}(12 \mathrm{mg}, 8 \%)$ as a yellow gum followed by dihydrobenzofuran $4 \mathrm{e}(54 \mathrm{mg}, 62 \%)$ as a colorless gum.

After $20 \mathrm{~h}$ the same procedure using $0.22 \mathrm{mmol}$ of oxetanol 1 afforded dihydrobenzofuran $4 \mathrm{e}(61 \mathrm{mg}, 81 \%)$.

Oxetane ether 3e: $\mathrm{R}_{f}=0.57$ (30\% EtOAc/hexane); $\mathrm{mp}=101-$ $104{ }^{\circ} \mathrm{C}$; IR (film) $/ \mathrm{cm}^{-1}$ 2951, 2875, 1611, 1508, 1455, 1291, 1231, 1179, 1112, 987, 813, 740, 698; ${ }^{1} \mathrm{H}$ NMR (400 MHz, $\left.\mathrm{CDCl}_{3}\right) \delta$ 7.52-7.30 (m, $\left.7 \mathrm{H}, 7 \times \mathrm{Ar}-\mathrm{CH}\right), 7.01-6.95(\mathrm{~m}, 4 \mathrm{H}, 4 \times$ Ar-CH), 6.46-6.40 (m, $2 \mathrm{H}, 2 \times \mathrm{Ar}-\mathrm{CH}), 5.09(\mathrm{~d}, J=7.3 \mathrm{~Hz}$, $\mathrm{C} H \mathrm{HOCHH}), 5.06\left(\mathrm{~s}, 2 \mathrm{H}, \mathrm{CH}_{2}\right), 4.90(\mathrm{~d}, J=7.3 \mathrm{~Hz}, 2 \mathrm{H}$, $\mathrm{CH} H \mathrm{OCH} H), 2.24\left(\mathrm{~s}, 3 \mathrm{H}, \mathrm{CH}_{3}\right) ;{ }^{13} \mathrm{C}$ NMR $\left(101 \mathrm{MHz}, \mathrm{CDCl}_{3}\right)$ 
$\delta 158.4\left(\mathrm{Ar}-C_{q^{-}} \mathrm{OBBn}\right), 152.6\left(\mathrm{Ar}_{(p-\mathrm{Me})} C_{q^{-}} \mathrm{O}\right), 136.8\left(\mathrm{Ph}-C_{q^{-}}\right.$ $\left.\mathrm{CH}_{2}\right), 132.6\left(\mathrm{Ar}-\mathrm{C}_{q}-\mathrm{C}_{\mathrm{q}}\right), 130.3\left(\mathrm{Ar}-\mathrm{C}_{q}-\mathrm{CH}_{3}\right), 129.8(2 \times \mathrm{Ar}-\mathrm{CH})$, $128.6(2 \times \mathrm{Ar}-\mathrm{CH}), 128.0(\mathrm{Ar}-\mathrm{CH}), 127.5(2 \times \mathrm{Ar}-\mathrm{CH}), 126.10(2$ $\times$ Ar-CH $), 116.0(2 \times$ Ar-CH $), 115.0(2 \times$ Ar-CH $), 82.9$ $\left(\mathrm{CH}_{2} \mathrm{OCH}_{2}\right), 80.0\left(\mathrm{C}_{\mathrm{q}}\right), 70.1\left(\mathrm{Ph}-\mathrm{CH}_{2}\right), 20.4\left(\mathrm{CH}_{3}\right)$; HRMS (TOFASAP+) $m / z$ calcd for $\mathrm{C}_{23} \mathrm{H}_{23} \mathrm{O}_{3}{ }^{+}[\mathrm{M}+\mathrm{H}]^{+}: 347.1647$, Found: 347.1650 .

Dimer 5e: $\mathrm{R}_{f}=0.42$ (30\% EtOAc/hexane); IR (film) $/ \mathrm{cm}^{-1} 3034$, 2872, 1610, 1511, 1490, 1455, 1382, 1299, 1243, 1182, 1130, $1025,982,830,812,735,698 ;{ }^{1} \mathrm{H}$ NMR $\left(400 \mathrm{MHz}, \mathrm{CDCl}_{3}\right)$ $\delta$ 7.48-7.32 (m, $10 \mathrm{H}, 10 \times \mathrm{Ar}-\mathrm{CH}), 7.29-7.20(\mathrm{~m}, 4 \mathrm{H}, 4 \times \mathrm{Ar}-$ $\mathrm{CH}), 7.00-6.97$ (m, 3 H, 3 × Ar-CH), 6.94-6.90 (m, $2 \mathrm{H}, 2 \times \mathrm{Ar}-$ $\mathrm{CH})$, 8.86-6.83 (m, 1 H, Ar-CH), 6.79-6.67 (m, 1 H, Ar-CH), 5.09 (s, $\left.2 \mathrm{H}, \mathrm{Ph}-\mathrm{CH}_{2}\right), 5.06$ (s, $\left.2 \mathrm{H}, \mathrm{Ph}-\mathrm{CH}_{2}\right), 4.91-4.69$ (m, $5 \mathrm{H}$, $\left.\mathrm{CH}_{2} \mathrm{OCH}_{2}+\mathrm{OCHHC}_{\mathrm{q}}\right), 4.55\left(\mathrm{~d}, J=9.0 \mathrm{~Hz}, 1 \mathrm{H}, \mathrm{OCH}_{\mathrm{C}} \mathrm{q}\right), 3.63$ $\left(\mathrm{d}, J=9.2 \mathrm{~Hz}, 1 \mathrm{H}, \mathrm{C}_{\mathrm{q}} \mathrm{OC} H \mathrm{HC}_{\mathrm{q}}\right), 3.51(\mathrm{~d}, J=9.2 \mathrm{~Hz}, 1 \mathrm{H}$, $\mathrm{C}_{\mathrm{q}} \mathrm{OCHHC}_{\mathrm{q}}$ ), $2.27\left(\mathrm{~s}, 3 \mathrm{H}, \mathrm{CH}_{3}\right) ;{ }^{13} \mathrm{C} \mathrm{NMR}\left(101 \mathrm{MHz}, \mathrm{CDCl}_{3}\right)$ $\delta 158.5\left(\mathrm{Ar}-\mathrm{C}_{\mathrm{q}}-\mathrm{OBn}\right), 158.0\left(\mathrm{Ar}-\mathrm{C}_{\mathrm{q}}-\mathrm{O}_{(\mathrm{DHBF})}\right), 157.5\left(\mathrm{Ar}^{-\mathrm{C}_{\mathrm{q}}}{ }^{-}\right.$ $\mathrm{OBn}), 137.0\left(\mathrm{Ph}-\mathrm{C}_{\mathrm{q}}\right), 136.7\left(\mathrm{Ph}-\mathrm{C}_{\mathrm{q}}\right), 135.6\left(\mathrm{Ar}_{(p-\mathrm{OBn})}-C_{q}-\mathrm{C}_{\mathrm{q}(\mathrm{DHBF})}\right)$, $131.7\left(\mathrm{Ar}-C_{q}-\mathrm{C}_{\mathrm{q}}\right), 131.6\left(\mathrm{Ar}-C_{q}-\mathrm{C}_{\mathrm{q}}\right), 129.8\left(\mathrm{Ar}-C_{q}-\mathrm{CH}_{3}\right), 129.3$ (Ar-CH), $128.62(2 \times$ Ar-CH), $128.58(2 \times \mathrm{Ar}-\mathrm{CH}), 128.3(2 \times$ Ar-CH), 128.1 (Ar-CH), $128.0(\mathrm{Ar}-\mathrm{CH}), 127.46(2 \times \mathrm{Ar}-\mathrm{CH})$, $127.45(2 \times \mathrm{Ar}-\mathrm{CH}), 127.44(2 \times \mathrm{Ar}-\mathrm{CH}), 125.8(\mathrm{Ar}-\mathrm{CH}), 114.9$ $(2 \times$ Ar- $\mathrm{CH}), \quad 114.6(2 \times$ Ar- $\mathrm{CH}), 109.5(\mathrm{Ar}-\mathrm{CH}), 81.8$

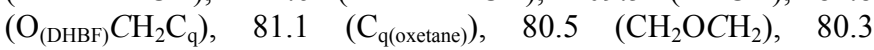
$\left(\mathrm{CH}_{2} \mathrm{OCH}_{2}\right), 70.1\left(\mathrm{Ph}-\mathrm{CH}_{2}\right), 70.0\left(\mathrm{Ph}-\mathrm{CH}_{2}\right), 68.3\left(\mathrm{C}_{\mathrm{q}} \mathrm{OCH}_{2} \mathrm{C}_{\mathrm{q}}\right)$, $54.0\left(\mathrm{C}_{\mathrm{q}(\mathrm{DHBF})}\right), 20.9\left(\mathrm{CH}_{3}\right)$; FTMS $(+\mathrm{pNSI}) \mathrm{m} / \mathrm{z}$ calcd for $\mathrm{C}_{39} \mathrm{H}_{40} \mathrm{O}_{5} \mathrm{~N}^{+}\left[\mathrm{M}+\mathrm{NH}_{4}\right]^{+}:$602.2901, Found: 602.2894.

Dihydrobenzofuran 4e: $\mathrm{R}_{f}=0.33(30 \%$ EtOAc/hexane); IR (film) $/ \mathrm{cm}^{-1} 3420$ (br, OH), 3034, 2868, 1608, 1580, 1489, 1454, $1381,1295,1241,1182,1127,1037,1024,971,827,809,732$, 696; ${ }^{1} \mathrm{H}$ NMR $\left(400 \mathrm{MHz}, \mathrm{CDCl}_{3}\right) \delta \quad 7.48-7.34(\mathrm{~m}, 5 \mathrm{H}, 5 \times \mathrm{Ar}-$ $\mathrm{CH}), 7.29-7.23(\mathrm{~m}, 2 \mathrm{H}, 2 \times \mathrm{Ar}-\mathrm{CH}), 7.07-6.96(\mathrm{~m}, 4 \mathrm{H}, 4 \times \mathrm{Ar}-$ $\mathrm{CH}), 6.81(\mathrm{~d}, J=8.1 \mathrm{~Hz}, 1 \mathrm{H}, 1 \times \mathrm{Ar}-\mathrm{CH}), 5.08\left(\mathrm{~s}, 2 \mathrm{H}, \mathrm{CH}_{2} \mathrm{Ph}\right)$, $4.76\left(\mathrm{~d}, J=8.9 \mathrm{~Hz}, \mathrm{OCHHC}_{\mathrm{q}}\right), 4.58\left(\mathrm{~d}, J=8.9 \mathrm{~Hz}, \mathrm{OCH} H C_{\mathrm{q}}\right)$, $4.12(\mathrm{dd}, J=11.0,3.9 \mathrm{~Hz}, \mathrm{CHHOH}), 4.04(\mathrm{dd}, J=11.0,7.5 \mathrm{~Hz}$, $\mathrm{CH} H \mathrm{OH}), 2.34\left(\mathrm{~s}, 3 \mathrm{H}, \mathrm{CH}_{3}\right), 1.58(\mathrm{~s}, 1 \mathrm{H}$, br. $\mathrm{OH}) ;{ }^{13} \mathrm{C} \mathrm{NMR}$ $\left(101 \mathrm{MHz}, \mathrm{CDCl}_{3}\right) \delta 158.6\left(\mathrm{Ar}-\mathrm{C}_{\mathrm{q}}-\mathrm{O}_{(\mathrm{DHBF})}\right), 157.6\left(\mathrm{Ar}-\mathrm{C}_{q}-\mathrm{OBn}\right)$, $136.8\left(\mathrm{Ph}-\mathrm{C}_{\mathrm{q}}\right), 135.0\left(\mathrm{Ar}-C_{q}-\mathrm{C}_{\mathrm{q}}\right), 130.1\left(\mathrm{Ar}_{(\mathrm{DHBF})}-C_{q}-\mathrm{C}_{\mathrm{q}}\right), 130.0$ $\left(\mathrm{Ar}-\mathrm{C}_{q}-\mathrm{CH}_{3}\right), 129.6(\mathrm{Ar}-\mathrm{CH}), 128.5(2 \times \mathrm{Ar}-\mathrm{CH}), 128.0(2 \times \mathrm{Ar}-$ $\mathrm{CH}), 127.9(\mathrm{Ar}-\mathrm{CH}), 127.4(2 \times \mathrm{Ar}-\mathrm{CH}), 125.5(\mathrm{Ar}-\mathrm{CH}), 115.0$ $(2 \times \mathrm{Ar}-\mathrm{CH}), 109.7(\mathrm{Ar}-\mathrm{CH}), 81.3\left(\mathrm{OCH}_{2} \mathrm{C}_{\mathrm{q}}\right), 70.0\left(\mathrm{OCH}_{2} \mathrm{Ph}\right)$, $67.5\left(\mathrm{CH}_{2} \mathrm{OH}\right), 55.7\left(\mathrm{C}_{\mathrm{q}}\right), 20.9\left(\mathrm{CH}_{3}\right)$; FTMS $(+\mathrm{pNSI}) \mathrm{m} / z$ calcd for $\mathrm{C}_{23} \mathrm{H}_{26} \mathrm{O}_{3} \mathrm{~N}^{+}\left[\mathrm{M}+\mathrm{NH}_{4}\right]^{+}$: 364.1907, Found: 364.1909. The observed spectroscopic data for this compound was consistent with that previously reported. ${ }^{[13]}$

\subsubsection{3-[4-(Benzyloxy)phenyl]-3-(4- methoxyphenoxy)oxetane $(\mathbf{3 f})$, \\ \{3-[4-(benzyloxy)phenyl]-5-methoxy-2,3-dihydro-1- benzofuran-3-yl\}methanol (4f) \\ and}

3-[4-(benzyloxy)phenyl]-3-[(\{3-[4-

(benzyloxy)phenyl]oxetan-3-yl\}oxy)methyl]-5methoxy-2,3-dihydro-1-benzofuran (5f)

Prepared according to General Procedure A described above, using 4-methoxyphenol (155 mg, $1.25 \mathrm{mmol})$ and a reaction time of $1 \mathrm{~h}$. Purification by flash chromatography $(30 \%$ EtOAc/hexane) afforded oxetane ether $3 f(10 \mathrm{mg}, 11 \%)$ as a white solid followed by dimer $\mathbf{5 f}(17 \mathrm{mg}, 19 \%)$ as a yellow gum followed by dihydrobenzofuran $\mathbf{4 f}(38 \mathrm{mg}, 42 \%)$ as a colorless gum.
After $20 \mathrm{~h}$ the same procedure afforded dihydrobenzofuran $4 \mathbf{f}$ (66 $\mathrm{mg}, 73 \%)$.

Oxetane ether 3f: $\mathrm{R}_{f}=0.43$ (30\% EtOAc/hexane); $\mathrm{mp}=100$ $102{ }^{\circ} \mathrm{C}$; IR (film) $/ \mathrm{cm}^{-1}$ 2952, 2876, 1610, 1504, 1455, 1381, $1295,1223,1178,1111,1035,985,906,825,730,698 ;{ }^{1} \mathrm{H}$ NMR $\left(400 \mathrm{MHz}, \mathrm{CDCl}_{3}\right) \delta$ 7.51-7.33 (m, $\left.7 \mathrm{H}, 7 \times \mathrm{Ar}-\mathrm{CH}\right), 7.02-6.96$ (m, $2 \mathrm{H}, 2 \times \mathrm{Ar}-\mathrm{CH}), 6.74-6.70(\mathrm{~m}, 2 \mathrm{H}, 2 \times \mathrm{Ar}-\mathrm{CH}), 6.50-6.44$ $(\mathrm{m}, 2 \mathrm{H}, 2 \times \mathrm{Ar}-\mathrm{CH}), 5.08(\mathrm{~d}, J=6.9 \mathrm{~Hz}, 2 \mathrm{H}, \mathrm{CHHOCHH}), 5.06$ (s, $2 \mathrm{H}, \mathrm{Ph}-\mathrm{CH}_{2}$ ), 4.89 (d, J=6.9 Hz, $2 \mathrm{H}, \mathrm{CH} H \mathrm{OCH} H$ ), 3.72 (s, $\left.3 \mathrm{H}, \mathrm{CH}_{3}\right) ;{ }^{13} \mathrm{C} \mathrm{NMR}\left(101 \mathrm{MHz}, \mathrm{CDCl}_{3}\right) \delta 158.4\left(\mathrm{Ar}-\mathrm{C}_{\mathrm{q}}-\mathrm{OBn}\right)$, $154.0\left(\mathrm{Ar}-\mathrm{C}_{\mathrm{q}}-\mathrm{OMe}\right), 148.7\left(\mathrm{Ar}-\mathrm{C}_{q}-\mathrm{O}-\mathrm{C}_{\mathrm{q}}\right), 136.8\left(\mathrm{Ph}-\mathrm{C}_{\mathrm{q}}-\mathrm{CH}_{2}\right)$, $132.6\left(\mathrm{Ar}-\mathrm{C}_{q}-\mathrm{C}_{\mathrm{q}}\right), 128.6(2 \times \mathrm{Ar}-\mathrm{CH}), 128.0(\mathrm{Ar}-\mathrm{CH}), 127.5(2 \times$ Ar-CH), $126.2(2 \times$ Ar-CH $), 117.2(2 \times$ Ar-CH $), 115.0(2 \times$ Ar$\mathrm{CH}), 114.6(2 \times \mathrm{Ar}-\mathrm{CH}), 82.8\left(\mathrm{CH}_{2} \mathrm{OCH}_{2}\right), 80.2\left(\mathrm{C}_{\mathrm{q}}\right), 70.1(\mathrm{Ph}-$ $\left.\mathrm{CH}_{2}\right)$, $55.6\left(\mathrm{CH}_{3}\right)$; FTMS (+pNSI) $\mathrm{m} / z$ calcd for $\mathrm{C}_{23} \mathrm{H}_{22} \mathrm{O}_{4} \mathrm{Na}^{+}$ $[\mathrm{M}+\mathrm{Na}]^{+}: 385.1410$, Found: 385.1412.

Dimer 5f: $\mathrm{R}_{f}=0.33$ (30\% EtOAc/hexane); IR (film) $/ \mathrm{cm}^{-1} 3034$, 2946, 2875, 1738, 1609, 1582, 1511, 1487, 1455, 1381, 1301, $1242,1201,1179,1026,982,908,830,734,698 ;{ }^{1} \mathrm{H}$ NMR $(400$ $\left.\mathrm{MHz}, \mathrm{CDCl}_{3}\right) \delta$ 7.50-7.34 (m, $\left.10 \mathrm{H}, 10 \times \mathrm{Ar}-\mathrm{CH}\right), 7.30-7.21$ (m, $4 \mathrm{H}, 4 \times \mathrm{Ar}-\mathrm{CH}), 7.05-7.00(\mathrm{~m}, 2 \mathrm{H}, 2 \times \mathrm{Ar}-\mathrm{CH}), 6.96-6.92$ (m, $2 \mathrm{H}, 2 \times \mathrm{Ar}-\mathrm{CH}), 6.82-6.77$ (m, $2 \mathrm{H}, 2 \times \mathrm{Ar}-\mathrm{CH}), 6.72-6.69$ (m, $1 \mathrm{H}, \mathrm{Ar}-\mathrm{CH}), 5.11\left(\mathrm{~s}, 2 \mathrm{H}, \mathrm{Ph}-\mathrm{CH}_{2}\right), 5.08\left(\mathrm{~s}, 2 \mathrm{H}, \mathrm{Ph}-\mathrm{CH}_{2}\right)$, $4.87(\mathrm{dd}, J=9.3,6.7 \mathrm{~Hz}, 2 \mathrm{H}, \mathrm{CHHOCHH}), 4.79(\mathrm{~d}, J=6.7 \mathrm{~Hz}$, $2 \mathrm{H}, \mathrm{CH} H \mathrm{OCH} H), 4.71\left(\mathrm{~d}, J=9.0 \mathrm{~Hz}, 1 \mathrm{H}, \mathrm{OCH} H \mathrm{C}_{\mathrm{q}(\text { oxetane })}\right)$, $4.53\left(\mathrm{~d}, J=9.0 \mathrm{~Hz}, 1 \mathrm{H}, \mathrm{OCH}_{\mathrm{q}} \mathrm{C}_{\text {(oxetane) }}\right), 3.76\left(\mathrm{~s}, 3 \mathrm{H}, \mathrm{CH}_{3}\right)$, $3.61\left(\mathrm{~d}, J=9.3 \mathrm{~Hz}, 1 \mathrm{H}, \mathrm{C}_{\mathrm{q}} \mathrm{OCHHC}\right.$ ), $3.56(\mathrm{~d}, J=9.3 \mathrm{~Hz}, 1 \mathrm{H}$, $\left.\mathrm{C}_{\mathrm{q}} \mathrm{OCH} H \mathrm{C}_{\mathrm{q}}\right) ;{ }^{13} \mathrm{C}$ NMR $\left(101 \mathrm{MHz}, \mathrm{CDCl}_{3}\right) \delta 158.5\left(\mathrm{Ar}-\mathrm{C}_{\mathrm{q}}{ }^{-}\right.$ $\mathrm{OBn}), 157.6\left(\mathrm{Ar}-\mathrm{C}_{\mathrm{q}}-\mathrm{OBn}\right), 154.2\left(\mathrm{Ar}-\mathrm{C}_{\mathrm{q}}-\mathrm{O}_{(\mathrm{DHBF})}\right.$ or $\left.\mathrm{Ar}-\mathrm{C}_{\mathrm{q}}-\mathrm{OMe}\right)$, 154.1 ( $\mathrm{Ar}-\mathrm{C}_{\mathrm{q}}-\mathrm{O}_{(\mathrm{DHBF})}$ or $\left.\mathrm{Ar}-\mathrm{C}_{\mathrm{q}}-\mathrm{OMe}\right), 136.9\left(\mathrm{Ph}-\mathrm{C}_{\mathrm{q}}\right), 136.7(\mathrm{Ph}-$ $\left.\mathrm{C}_{\mathrm{q}}\right), 135.3\left(\mathrm{Ar}_{(p-\mathrm{OBn})^{-}} C_{q^{-}}-\mathrm{C}_{\mathrm{q}(\mathrm{DHBF})}\right), 131.6\left(\mathrm{Ar}-C_{q^{-}}-\mathrm{C}_{\mathrm{q}}\right), 131.5\left(\mathrm{Ar}-C_{q^{-}}\right.$ $\left.\mathrm{C}_{\mathrm{q}}\right), 128.62(2 \times \mathrm{Ar}-\mathrm{CH}), 128.58(2 \times \mathrm{Ar}-\mathrm{CH}), 128.3(2 \times \mathrm{Ar}-$ $\mathrm{CH}), 128.1(\mathrm{Ar}-\mathrm{CH}), 128.0(\mathrm{Ar}-\mathrm{CH}), 127.5(2 \times \mathrm{Ar}-\mathrm{CH}), 127.4$ $(4 \times \mathrm{Ar}-\mathrm{CH}), 114.9(2 \times \mathrm{Ar}-\mathrm{CH}), 114.6(2 \times \mathrm{Ar}-\mathrm{CH}), 114.1(\mathrm{Ar}-$ $\mathrm{CH}), 111.4$ (Ar-CH), 109.9 (Ar-CH), $82.0\left(\mathrm{O}_{(\mathrm{DHBF})} \mathrm{CH}_{2} \mathrm{C}_{\mathrm{q}}\right), 81.2$ $\left(\mathrm{C}_{\mathrm{q} \text { (oxetane) })}\right), \quad 80.3 \quad\left(\mathrm{CH}_{2} \mathrm{OCH}_{2}\right), \quad 70.1 \quad\left(2 \times \mathrm{Ph}_{-} \mathrm{CH}_{2}\right), 68.2$ $\left(\mathrm{C}_{\mathrm{q}} \mathrm{OCH}_{2} \mathrm{C}_{\mathrm{q}}\right), 56.0\left(\mathrm{CH}_{3}\right), 54.4\left(\mathrm{C}_{\mathrm{q}(\mathrm{DHBF})}\right)$; FTMS $(+\mathrm{pNSI}) \mathrm{m} / \mathrm{z}$ calcd for $\mathrm{C}_{39} \mathrm{H}_{40} \mathrm{O}_{6} \mathrm{~N}^{+}\left[\mathrm{M}+\mathrm{NH}_{4}\right]^{+}:$618.2850, Found: 618.2843.

Dihydrobenzofuran 4f: $\mathrm{R}_{f}=0.18$ (30\% EtOAc/hexane); IR (film) $/ \mathrm{cm}^{-1} 3442$ (br, OH), 3034, 2941, 1608, 1510, 1486, 1466, $1381,1242,1199,1179,1025,974,907,828,804,727,696 ;{ }^{1} \mathrm{H}$ NMR $\left(400 \mathrm{MHz}, \mathrm{CDCl}_{3}\right) \delta$ 7.48-7.34 (m, $\left.5 \mathrm{H}, 5 \times \mathrm{Ar}-\mathrm{CH}\right)$, 7.31-7.21 (m, 2 H, $2 \times$ Ar-CH), 7.02-6.92 (m, 2 H, $2 \times$ Ar-CH), 6.86-6.76 (m, $3 \mathrm{H}, 3 \times \mathrm{Ar}-\mathrm{CH}), 5.08\left(\mathrm{~s}, 2 \mathrm{H}, \mathrm{PhCH}_{2}\right), 4.75$ (d, $J$ $\left.=8.9 \mathrm{~Hz}, 1 \mathrm{H}, \mathrm{OCHHC}_{\mathrm{q}}\right), 4.56\left(\mathrm{~d}, J=8.9 \mathrm{~Hz}, 1 \mathrm{H}, \mathrm{OCH} H \mathrm{C}_{\mathrm{q}}\right)$, $4.13(\mathrm{dd}, J=11.2,3.3 \mathrm{~Hz}, 1 \mathrm{H}, \mathrm{CH} H O H), 4.05(\mathrm{dd}, J=11.2,7.1$ $\mathrm{Hz}, 1 \mathrm{H}, \mathrm{CH} H \mathrm{OH}), 3.79\left(\mathrm{~s}, 3 \mathrm{H}, \mathrm{CH}_{3}\right) ;{ }^{13} \mathrm{C} \mathrm{NMR} \mathrm{(101} \mathrm{MHz,}$ $\left.\mathrm{CDCl}_{3}\right) \delta 157.8\left(\mathrm{Ar}_{-} \mathrm{C}_{\mathrm{q}}-\mathrm{OBn}\right), 154.8\left(\mathrm{Ar}_{(\mathrm{DHBF})}-C_{q}-\mathrm{OCH}_{2}\right), 154.3$ $\left.\left(\mathrm{Ar}-C_{q}-\mathrm{OCH}_{3}\right), 136.9\left(\mathrm{Ph}-\mathrm{C}_{\mathrm{q}}\right), 134.7 \quad\left(\mathrm{Ar}_{(p-\mathrm{OBn}}\right)^{-} C_{q}-\mathrm{C}_{\mathrm{q}}\right), 131.2$ $\left(\mathrm{Ar}_{(\mathrm{DHBF})} \mathrm{C}_{q}-\mathrm{C}_{\mathrm{q}}\right), 128.6(2 \times \mathrm{Ar}-\mathrm{CH}), 128.1(2 \times \mathrm{Ar}-\mathrm{CH}), 128.0$ $(\mathrm{Ar}-\mathrm{CH}), 127.4(2 \times \mathrm{Ar}-\mathrm{CH}), 115.1(2 \times \mathrm{Ar}-\mathrm{CH}), 114.2(\mathrm{Ar}-\mathrm{CH})$, $111.2(\mathrm{Ar}-\mathrm{CH}), 110.1(\mathrm{Ar}-\mathrm{CH}), 81.5\left(\mathrm{OCH}_{2} \mathrm{C}_{\mathrm{q}}\right), 70.0\left(\mathrm{Ph}-\mathrm{CH}_{2}\right)$, $67.4\left(\mathrm{CH}_{2} \mathrm{OH}\right), 56.1\left(\mathrm{C}_{\mathrm{q}}\right), 56.0\left(\mathrm{CH}_{3}\right)$; FTMS $(+\mathrm{pNSI}) \mathrm{m} / z$ calcd for $\mathrm{C}_{23} \mathrm{H}_{26} \mathrm{O}_{4} \mathrm{~N}^{+}\left[\mathrm{M}+\mathrm{NH}_{4}\right]^{+}$: 380.1856, Found: 380.1857. The observed spectroscopic data for this compound was consistent with that previously reported. ${ }^{[13]}$

\subsection{Reaction profile experiments:}

Oxetanol 1 (64 mg, $0.25 \mathrm{mmol})$, phenol 2d,f,g (1.25 mmol, 5 equiv), tetrabutylammonium hexafluorophosphate $(5.3 \mathrm{mg}$, $0.0138 \mathrm{mmol})$, mesitylene $(12.8 \mathrm{mg}, 0.10 \mathrm{mmol})$ and lithium bis(trifluoromethanesulfonimide) $(7.9 \mathrm{mg}, 0.0275 \mathrm{mmol})$ were added into an NMR tube. Immediately prior to starting the ${ }^{1} \mathrm{H}$ 
NMR experiments, $\mathrm{CDCl}_{3}(0.5 \mathrm{~mL})$ was added and the NMR tube inverted 3 times to mix the reagents. ${ }^{1} \mathrm{H}$ NMR spectra were recorded on a $400 \mathrm{MHz}$ spectrometer at $40{ }^{\circ} \mathrm{C}$ with a spectrum recorded every 30 seconds for the first hour and then every $5 \mathrm{~min}$ thereafter until the reaction reached completion. Yields were calculated from the $1 \mathrm{H}$ NMR spectrum by comparison to mesitylene as the internal standard, using the following signals: mesitylene $(2.26 \mathrm{ppm}, 9 \mathrm{H})$, oxetanol $1(4.90,2 \mathrm{H})$ oxetane ether 3 (6.56-6.44 ppm, $2 \mathrm{H})$, dihydrobenzofuran 4 (4.56-4.58 ppm, 1 $\left.\mathrm{H}, \mathrm{OCH} \mathrm{C}_{\mathrm{q}}\right)$, dimer $5(4.71 \mathrm{ppm}, 1 \mathrm{H})$, diaryloxetane $6(5.25,4$ $\mathrm{H})$.

\subsubsection{Reaction of oxetanol 1 with phenol $\mathbf{2 g}$}

Reaction set up as described in section 4.4., using phenol $\mathbf{2 g}$ as the nucleophile. The diaryloxetane and dihydrobenzofuran were identified in the ${ }^{1} \mathrm{H}$ NMR study through comparison of purified samples with previously reported spectroscopic data. ${ }^{[13]}$

Oxetane ether 3g: See section 4.7.

Dihydrobenzofuran 4g: $\mathrm{R}_{f}=0.30$ (25\% EtOAc/hexane); IR (film) $/ \mathrm{cm}^{-1} 3462$ (br. OH), 2882, 1609, 1511, 1481, 1458, 1382, 1239, 1184, 1123, 1017, 973, 827, 753, 698; ${ }^{1} \mathrm{H}$ NMR (400 MHz, $\left.\mathrm{CDCl}_{3}\right) \delta$ 7.48-7.30 (m, $\left.5 \mathrm{H}, 5 \times \mathrm{Ar}-\mathrm{CH}\right), 7.30-7.17(\mathrm{~m}, 4 \mathrm{H}, 4 \times$ Ar- $\mathrm{CH}), 7.00-6.84$ (m, $4 \mathrm{H}, 4 \times \mathrm{Ar}-\mathrm{CH}), 5.06$ (s, $\left.2 \mathrm{H}, \mathrm{OCH}_{2} \mathrm{Ph}\right)$, $4.77\left(\mathrm{~d}, J=8.9 \mathrm{~Hz}, 1 \mathrm{H}, \mathrm{OCHHC}_{\mathrm{q}}\right), 4.58(\mathrm{~d}, J=8.9 \mathrm{~Hz}, 1 \mathrm{H}$, $\left.\mathrm{OCH} H C_{\mathrm{q}}\right), 4.12(\mathrm{~d}, J=11.1 \mathrm{~Hz}, 1 \mathrm{H}, \mathrm{CHHOH}), 4.04(\mathrm{~d}, J=11.1$ $\mathrm{Hz}, 1 \mathrm{H}, \mathrm{CH} H \mathrm{OH}) ;{ }^{13} \mathrm{C} \mathrm{NMR}\left(101 \mathrm{MHz}, \mathrm{CDCl}_{3}\right) \delta 160.8$ $\left(\mathrm{Ar}_{(\mathrm{DHBF})} \mathrm{C}_{q}-\mathrm{OCH}_{2}\right), 157.8\left(\mathrm{Ar}-\mathrm{C}_{\mathrm{q}}-\mathrm{OBn}\right), 136.9\left(\mathrm{Ph}-\mathrm{C}_{\mathrm{q}}\right), 134.9$ $\left(\mathrm{Ar}-C_{q}-\mathrm{C}_{\mathrm{q}}\right), 130.1\left(\mathrm{Ar}_{(\mathrm{DHBF})}-C_{q}-\mathrm{C}_{\mathrm{q}}\right), 129.3(\mathrm{Ar}-\mathrm{CH}), 128.6(2 \times$ Ar- $\mathrm{CH}), 128.1(2 \times \mathrm{Ar}-\mathrm{CH}), 128.0$ (Ar-CH), $127.5(2 \times \mathrm{Ar}-\mathrm{CH})$, $125.2(\mathrm{Ar}-\mathrm{CH}), 120.8(\mathrm{Ar}-\mathrm{CH}), 115.1(2 \times \mathrm{Ar}-\mathrm{CH}), 110.2(\mathrm{Ar}-$ $\mathrm{CH}), 81.3\left(\mathrm{OCH}_{2} \mathrm{C}_{\mathrm{q}}\right), 70.0\left(\mathrm{PhCH}_{2}\right), 67.7\left(\mathrm{CH}_{2} \mathrm{OH}\right), 55.8\left(\mathrm{C}_{\mathrm{q}}\right)$; HRMS $\left(\mathrm{CI}^{+}\right) \mathrm{m} / \mathrm{z}$ calcd for $\mathrm{C}_{22} \mathrm{H}_{24} \mathrm{NO}_{3}{ }^{+}\left[\mathrm{M}+\mathrm{NH}_{4}\right]^{+}: 350.1756$, Found: 350.1767

Diaryloxetane 6g: $\mathrm{R}_{f}=0.23(25 \%$ EtOAc/hexane $) ; \mathrm{mp}=164$ $166{ }^{\circ} \mathrm{C}$; IR (film) $/ \mathrm{cm}^{-1} 3325$ (br. OH), 2881, 2045, 1608, 1591, $1511,1455,1433,1384,1269,1228,1207,1176,1111,1015$, 973, 930, 836, 806, 741, 696; ${ }^{1} \mathrm{H}$ NMR (400 MHz, $\left.\mathrm{CDCl}_{3}\right) \delta$ 7.49-7.30 (m, $5 \mathrm{H}, 5 \times \mathrm{Ar}-\mathrm{CH}), 7.17-7.11(\mathrm{~m}, 2 \mathrm{H}, 2 \times \mathrm{Ar}-\mathrm{CH})$, 7.11-7.05 (m, $2 \mathrm{H}, 2 \times \mathrm{Ar}-\mathrm{CH}), 7.01-6.94\left(\mathrm{~m}, 2 \mathrm{H}, 2 \times \mathrm{Ar}_{\text {(phenol) }}{ }^{-}\right.$ $\mathrm{CH}), 6.85-6.78\left(\mathrm{~m}, 2 \mathrm{H}, 2 \times \mathrm{Ar}_{(\text {phenol) }}-\mathrm{CH}\right), 5.21(\mathrm{~s}, 4 \mathrm{H}$, $\left.\mathrm{CH}_{2} \mathrm{OCH}_{2}\right), 5.15(\mathrm{~s}, 1 \mathrm{H}, \mathrm{OH}), 5.07\left(\mathrm{~s}, 2 \mathrm{H}, \mathrm{CH}_{2} \mathrm{Ph}\right) ;{ }^{13} \mathrm{C} \mathrm{NMR}$ $\left(101 \mathrm{MHz}, \mathrm{CDCl}_{3}\right) \delta 157.4\left(\mathrm{Ar}_{-} \mathrm{C}_{\mathrm{q}}-\mathrm{OBn}\right), 154.3\left(\mathrm{Ar}-\mathrm{C}_{\mathrm{q}}-\mathrm{OH}\right)$, $138.4\left(\mathrm{Ar}_{(\mathrm{phenol})}-C_{q}-\mathrm{C}_{\mathrm{q}}\right), 138.2\left(\mathrm{Ar}_{(p-\mathrm{OBn})}-C_{q}-\mathrm{C}_{\mathrm{q}}\right), 136.9\left(\mathrm{Ph}-\mathrm{C}_{\mathrm{q}}\right)$, $128.6(2 \times \mathrm{Ar}-\mathrm{CH}), 128.0(\mathrm{Ar}-\mathrm{CH}), 127.8(2 \times \mathrm{Ar}-\mathrm{CH}), 127.6(2$ $\times \mathrm{Ar}-\mathrm{CH}), 127.5(2 \times \mathrm{Ar}-\mathrm{CH}), 115.3\left(2 \times \mathrm{Ar}_{(\text {phenol })}-\mathrm{CH}\right), 114.8(2$ $\left.\times \mathrm{Ar}_{\text {(phenol) }}-\mathrm{CH}\right), 85.0\left(\mathrm{CH}_{2} \mathrm{OCH}_{2}\right), 70.1\left(\mathrm{CH}_{2} \mathrm{Ph}\right), 50.3\left(\mathrm{C}_{\mathrm{q}}\right)$; HRMS $\left(\mathrm{CI}^{+}\right) \mathrm{m} / \mathrm{z}$ calcd for $\mathrm{C}_{22} \mathrm{H}_{24} \mathrm{NO}_{3}{ }^{+}\left[\mathrm{M}+\mathrm{NH}_{4}\right]^{+}: 350.1756$, Found: 350.1772 .

4.5. [4-(Benzyloxy)phenyl]-3-(4-

fluorophenoxy)oxetane (3d)

Oxetanol 1 (64 mg, $0.25 \mathrm{mmol})$, 4-fluorophenol (140 mg, $1.25 \mathrm{mmol})$, tetrabutylammonium hexafluorophosphate $(5.3 \mathrm{mg}$, $0.0138 \mathrm{mmol})$, lithium bis(trifluoromethanesulfonimide) $(7.9 \mathrm{mg}$, $0.0275 \mathrm{mmol})$ and $\mathrm{CHCl}_{3}(0.5 \mathrm{~mL})$ were added into a ${ }^{1} \mathrm{H}$ NMR tube. The reaction mixture was stirred at $40{ }^{\circ} \mathrm{C}$ for $10 \mathrm{~min}$ then quenched with sat. aq. $\mathrm{NaHCO}_{3}$. The layers were separated and the aqueous portion extracted with dichloromethane $(3 \times 5 \mathrm{~mL})$. The organic extracts were combined, washed with aq. $\mathrm{NaOH}(1$ $\mathrm{M}, 15 \mathrm{~mL}$ ) then dried over $\mathrm{Na}_{2} \mathrm{SO}_{4}$, filtered and concentrated in vacuo. Purification by flash chromatography $\left(15 \% \mathrm{Et}_{2} \mathrm{O} /\right.$ pentane $)$ afforded oxetane ether $\mathbf{3 d}(49 \mathrm{mg}, 55 \%)$ as a white solid. For data see 4.3.4.

\subsection{Synthesis of $3 \boldsymbol{d}$ on a $1.6 \mathrm{mmol}$ scale}

Oxetanol 1 (410 mg, $1.6 \mathrm{mmol}$ ), 4-fluorophenol (897 mg, 8.0 mmol), tetrabutylammonium hexafluorophosphate (34 mg, 0.088 $\mathrm{mmol}$ ), lithium bis(trifluoromethanesulfonimide) (51 mg, 0.176 mmol) and $\mathrm{CHCl}_{3}(3.2 \mathrm{~mL})$ were added into a round bottomed flask. The reaction mixture was stirred at $40{ }^{\circ} \mathrm{C}$ for $20 \mathrm{~min}$ then quenched with sat. aq. $\mathrm{NaHCO}_{3}(10 \mathrm{~mL})$. The layers were separated and the aqueous portion extracted with dichloromethane $(3 \times 15 \mathrm{~mL})$. The organic extracts were combined, washed with aq. $\mathrm{NaOH}(1 \mathrm{M}, 20 \mathrm{~mL})$ then dried over $\mathrm{Na}_{2} \mathrm{SO}_{4}$, filtered and concentrated in vacuo. Purification by flash chromatography $\left(15 \% \mathrm{Et}_{2} \mathrm{O} /\right.$ pentane) afforded oxetane ether $\mathbf{3 d}$ $(283 \mathrm{mg}, 50 \%)$ as a white solid. For data see 4.3.4.

\subsection{3-[4-(Benzyloxy)phenyl]-3-(4- methoxyphenoxy) oxetane (3f)}

Oxetanol 1 (64 mg, $0.25 \mathrm{mmol})$, 4-methoxyphenol (155 mg, $1.25 \mathrm{mmol})$, tetrabutylammonium hexafluorophosphate $(5.3 \mathrm{mg}$, $0.0138 \mathrm{mmol})$, Lithium bis(trifluoromethanesulfonimide) $(7.9 \mathrm{mg}, 0.0275 \mathrm{mmol})$ and $\mathrm{CHCl}_{3}(0.5 \mathrm{~mL})$ were added into a ${ }^{1} \mathrm{H}$ NMR tube. The reaction mixture was stirred at $40{ }^{\circ} \mathrm{C}$ for 15 minutes then quenched with sat. aq. $\mathrm{NaHCO}_{3}$. The layers were separated and the aqueous portion extracted with dichloromethane $(3 \times 5 \mathrm{~mL})$. The organic extracts were combined, washed with aq. $\mathrm{NaOH}(1 \mathrm{M}, 15 \mathrm{~mL})$ then dried over $\mathrm{Na}_{2} \mathrm{SO}_{4}$, filtered and concentrated in vacuo. Purification by flash chromatography (15\% EtOAc/hexane) afforded oxetane ether $\mathbf{3 f}$ (43 mg, 47\%) as a white solid. For data see 4.3.6.

\subsection{3-(4-(Benzyloxy)phenyl)-3-phenoxyoxetane (3g)}

Oxetanol 1 (64 mg, $0.25 \mathrm{mmol})$, phenol (118 mg, $1.25 \mathrm{mmol}$ ), tetrabutylammonium hexafluorophosphate $(5.3 \mathrm{mg}, \quad 0.0138$ $\mathrm{mmol}$ ), lithium bis(trifluoromethanesulfonimide) (7.9 mg, 0.0275 mmol) and $\mathrm{CHCl}_{3}(0.5 \mathrm{~mL})$ were added into a ${ }^{1} \mathrm{H}$ NMR tube. The reaction mixture was stirred at $40{ }^{\circ} \mathrm{C}$ for $12 \mathrm{~min}$ then quenched with sat. aq. $\mathrm{NaHCO}_{3}$. The layers were separated and the aqueous portion extracted with dichloromethane $(3 \times 5 \mathrm{~mL})$. The organic extracts were combined, washed with aq. $\mathrm{NaOH}(1 \mathrm{M}, 15 \mathrm{~mL})$ then dried over $\mathrm{Na}_{2} \mathrm{SO}_{4}$, filtered and concentrated in vacuo. Purification by flash chromatography $(10 \%$ EtOAc/hexane $)$ afforded oxetane ether $\mathbf{3 g}(23 \mathrm{mg}, 28 \%)$ as a white solid. $\mathrm{R}_{f}=$ $0.24(10 \%$ EtOAc/hexane $) ; \mathrm{mp}=113-114{ }^{\circ} \mathrm{C}$; IR (film) $/ \mathrm{cm}^{-1}$ 3036, 2950, 2877, 1611, 1587, 1512, 1489, 1455, 1382, 1297, $1232,1179,1113,1076,1025,987,831,752,694 ;{ }^{1} \mathrm{H}$ NMR $(400$ $\left.\mathrm{MHz}, \mathrm{CDCl}_{3}\right) \delta$ 7.52-7.33 (m, $\left.7 \mathrm{H}, 7 \times \mathrm{Ar}-\mathrm{CH}\right), 7.21-6.15(\mathrm{~m}$, $2 \mathrm{H}, 2 \times \mathrm{Ar}-\mathrm{CH}), 7.02-6.98(\mathrm{~m}, 2 \mathrm{H}, 2 \times \mathrm{Ar}-\mathrm{CH}), 6.94-6.90(\mathrm{~m}$, $1 \mathrm{H}, \mathrm{Ar}-\mathrm{CH}), 6.56-6.50$ (m, $2 \mathrm{H}, 2 \times \mathrm{Ar}-\mathrm{CH}), 5.11(\mathrm{~d}, J=7.4 \mathrm{~Hz}$, $2 \mathrm{H}, \mathrm{CHHOCHH}), 5.06\left(\mathrm{~s}, 2 \mathrm{H}, \mathrm{Ph}-\mathrm{CH}_{2}\right), 4.92(\mathrm{~d}, J=7.4 \mathrm{~Hz}, 2$ $\mathrm{H}, \mathrm{CH} H \mathrm{OCH} H) ;{ }^{13} \mathrm{C}$ NMR $\left(101 \mathrm{MHz}, \mathrm{CDCl}_{3}\right) \delta 158.4\left(\mathrm{Ar}_{-} \mathrm{C}_{\mathrm{q}}-\right.$ $\mathrm{OBn}), 154.8\left(\mathrm{Ar}-\mathrm{C}_{\mathrm{q}}-\mathrm{OC}_{\mathrm{q}}\right), 136.8\left(\mathrm{Ph}-\mathrm{C}_{\mathrm{q}}-\mathrm{CH}_{2}\right), 132.4\left(\mathrm{Ar}-\mathrm{C}_{q}-\mathrm{C}_{\mathrm{q}}\right)$, $129.4(2 \times \mathrm{Ar}-\mathrm{CH}), 128.6(2 \times \mathrm{Ar}-\mathrm{CH}), 128.0(\mathrm{Ar}-\mathrm{CH}), 127.5(2$ $\times$ Ar-CH $), 126.1(2 \times$ Ar-CH $), 121.0(\mathrm{Ar}-\mathrm{CH}), 116.2(2 \times \mathrm{Ar}-$ $\mathrm{CH}), 115.1(2 \times \mathrm{Ar}-\mathrm{CH}), 82.9\left(\mathrm{CH}_{2} \mathrm{OCH}_{2}\right), 80.1\left(\mathrm{C}_{\mathrm{q}}\right), 70.1(\mathrm{Ph}-$ $\mathrm{CH}_{2}$ ); HRMS (ESI-TOF) $\mathrm{m} / \mathrm{z}$ calcd for $\mathrm{C}_{22} \mathrm{H}_{21} \mathrm{O}_{3}{ }^{+}[\mathrm{M}+\mathrm{H}]^{+}$: 333.1491, Found: 333.1497.

\subsection{Synthesis of $\mathbf{3}$ from $3 \boldsymbol{d}$}

Lithium bis(trifluoromethanesulfonimide) (6.3 $\mathrm{mg}$, $0.022 \mathrm{mmol}$ ) and tetrabutylammonium hexafluorophosphate (4.3 $\mathrm{mg}, 0.011 \mathrm{mmol})$ were added to a solution of oxetane ether 3d $(70 \mathrm{mg}, 0.20 \mathrm{mmol})$ and 4-methoxyphenol (124 mg, $1.00 \mathrm{mmol})$ in chloroform $(0.4 \mathrm{~mL})$. The reaction mixture was 
stirred at $40{ }^{\circ} \mathrm{C}$ for $20 \mathrm{~h}$ then quenched with sat. aq. $\mathrm{NaHCO}_{3}$. The layers were separated and the aqueous portion extracted with dichloromethane $(3 \times 5 \mathrm{~mL})$. The organic extracts were combined, washed with aq. $\mathrm{NaOH}(1 \mathrm{M}, 15 \mathrm{~mL})$ then dried over $\mathrm{Na}_{2} \mathrm{SO}_{4}$, filtered and concentrated in vacuo. Purification by flash chromatography $\left(50 \% \mathrm{Et}_{2} \mathrm{O} /\right.$ pentane) afforded oxetane ether $\mathbf{3 f}$ (50 mg, 55\%) as a white solid. For data see 4.3.6.

\section{Acknowledgments}

We gratefully acknowledge The Royal Society [University Research Fellowship (to J.A.B.); URF appointed grant], the EPSRC [CAF to J.A.B. (EP/J001538/1); Impact Acceleration Account (EP/K503733/1)], and Imperial College London for a President's Scholarship (to R.A.C). We acknowledge the EPSRC National Mass Spectrometry Facility, Swansea.

\section{References and notes}

[1] C. Friedel, J. M. Crafts, J. Chem. Soc. 1877, 32, 725.

[2] For recent reviews on catalytic C-O activation, see: a) M. Dryzhakov, E. Richmond, J. Moran, Synthesis 2016, 48, 935. b) M. Rueping, B. J. Nachtsheim, Beilstein J. Org. Chem., 2010, 6, 6. c) L. Chen, X.-P. Yin, C.-H. Wang, J. Zhou, Org. Biomol. Chem. 2014, 12, 6033.

[3] For selected leading references, see: a) M. Niggemann, M. J. Meel, Angew. Chem. Int. Ed. 2010, 49, 3684; Angew. Chem. 2010, 122, 3767. b) M. Dryzhakov, M. Hellal, E. Wolf, F. C. Falk, J. Moran, J. Am. Chem. Soc. 2015, 137, 9555. c) S. Haubenreisser, M. Niggemann, $A d v$. Synth. Catal. 2011, 353, 469.

[4] a) I. Y. Chukicheva, I. V. Fedorova, A. A. Koroleva, A. V. Kuchin, Chem. Nat. Compd. 2008, 44, 450. b) B. M. Devassy, S. B. Halligudi, S. G. Hegde, A. B. Halgeri, F. Lefebvre, Chem. Commun. 2002, 1074. c) G. Li, B. Wang, J. Wang, L. Yan, J. Suo, J. Chem. Res 2005, 173. d) M. Ghiaci, B. Aghabarari, Chinese J. Catal. 2010, 31, 759.

[5] W. K. Gray, F. R. Smail, M. G. Hitzler, S. K. Ross, M. Poliakoff, J. Am. Chem. Soc. 1999, 121, 10711.

[6] F. Adam, K. M. Hello, T. H. Ali, Appl. Catal. A Gen. 2011, 399, 42.

[7] a) J. A. Bull, R. A. Croft, O. A. Davis, R. Doran, K. F. Morgan, Chem. Rev. 2016, 116, 12150. b) J. A. Burkhard, G. Wuitschik, M. RogersEvans, K. Müller, E. M. Carreira, Angew. Chem. Int. Ed. 2010, 49, 9052.

[8] For oxetane containing fragments see: a) K. F. Morgan, I. A. Hollingsworth, J. A. Bull, Chem. Commun. 2014, 50, 5203. b) O. A. Davis, J. A. Bull, Angew. Chem. Int. Ed. 2014, 53, 14230. c) O. A. Davis, R. A. Croft, J. A. Bull, Chem. Commun. 2015, 51, 15446. d) K. F. Morgan, I. A. Hollingsworth, J. A. Bull, Org. Biomol. Chem. 2015, 13, 5265 .

[9] For selected examples in medicinal chemistry, see: a) A. F. Stepan, K. Karki, W. S. McDonald, P. H. Dorff, J. K. Dutra, K. J. DiRico, A. Won, C. Subramanyam, I. V Efremov, C. J. O'Donnell, et al., J. Med. Chem. 2011, 54, 7772. b) Z. Song, Y. Yang, Z. Liu, X. Peng, J. Guo, X. Yang, K. Wu, J. Ai, J. Ding, M. Geng, A. Zhang, J. Med. Chem. 2015, 58, 197.

[10] G. Wuitschik, M. Rogers-Evans, K. Müller, H. Fischer, B. Wagner, F. Schuler, L. Polonchuk, E. M. Carreira, Angew. Chem. Int. Ed. 2006, 45, 7736; Angew. Chem. 2006, 118, 7900.

[11] P. Mukherjee, M. Pettersson, J. K. Dutra, L. Xie, C. W. am Ende, ChemMedChem 2017, 12, 1574.

[12] a) G. Wuitschik, M. Rogers-Evans, A. Buckl, M. Bernasconi, M. Märki, T. Godel, H. Fischer, B. Wagner, I. Parrilla, F. Schuler, et al., Angew. Chem. Int. Ed. 2008, 47, 4512.; Angew. Chem. 2008, 120, 4588. b) G. Wuitschik, E. M. Carreira, B. Wagner, H. Fischer, I. Parrilla, F. Schuler, M. Rogers-Evans, K. Müller, J. Med. Chem. 2010, 53, 3227. c) J. A. Burkhard, G. Wuitschik, J.-M. Plancher, M. RogersEvans, E. M. Carreira, Org. Lett. 2013, 15, 4312.

[13] R. A. Croft, J. J. Mousseau, C. Choi, J. A. Bull, Chem. Eur. J. 2016, $22,16271$.
[14] For peptide isosteres: a) M. McLaughlin, R. Yazaki, T. C. Fessard, E. M. Carreira, Org. Lett. 2014, 16, 4070. b) N. H. Powell, G. J. Clarkson, R. Notman, P. Raubo, N. G. Martin, M. Shipman, Chem. Commun. 2014, 50, 8797. c) J. D. Beadle, A. Knuhtsen, A. Hoose, P. Raubo, A. G. Jamieson, M. Shipman, Org. Lett. 2017, 19, 3303. d) G. P. Möller, S. Müller, B. T. Wolfstädter, S. Wolfrum, D. Schepmann, B. Wünsch, E. M. Carreira, Org. Lett. 2017, 19, 2510.

[15] R. A. Croft, J. J. Mousseau, C. Choi, J. A. Bull, Chem. Eur. J. 2018, 24, 818-821

[16] P. Lassalas, K. Oukoloff, V. Makani, M. James, V. Tran, Y. Yao, L. Huang, K. Vijayendran, L. Monti, J. Q. Trojanowski, V. M.-Y. Lee, M. C. Kozlowski, A. B. Smith, III, K. R. Brunden, C. Ballatore, ACS Med. Chem. Lett. 2017, 8, 864.

[17] C. A. Malapit, A. R. Howell, J. Org. Chem. 2015, 80, 8489.

[18] R. Sharma, S. Halder, S. Kumar, Piramal Enterprises Ltd. WO2014/170842 A2, 2014. 